\title{
p53 and miR-34a Feedback Promotes Lung Epithelial Injury and Pulmonary Fibrosis
}

Shwetha K. Shetty, ${ }^{*}$ Nivedita Tiwari, ${ }^{*}$ Amarnath S. Marudamuthu, ${ }^{*}$ Bijesh Puthusseri, ${ }^{*}$ Yashodhar P. Bhandary, ${ }^{*}$ Jian Fu, Jeffrey Levin, ${ }^{\ddagger}$ Steven Idell, ${ }^{*}$ and Sreerama Shetty*

From the Department of Medicine, * Texas Lung Injury Institute, and the Division of Occupational Medicine, ${ }^{\ddagger}$ Department of Medicine, University of Texas Health Science Center at Tyler, Tyler, Texas; and the Center for Research on Environmental Disease and Toxicology, ${ }^{\dagger}$ College of Medicine, University of Kentucky, Lexington, Kentucky

\author{
Accepted for publication \\ December 27, 2016. \\ Address correspondence to \\ Sreerama Shetty, Ph.D., \\ Department of Medicine, Texas \\ Lung Injury Institute, Univer- \\ sity of Texas Health Science \\ Center at Tyler, Tyler, \\ TX 75708. E-mail: sreerama. \\ shetty@uthct.edu.
}

\begin{abstract}
Idiopathic pulmonary fibrosis (IPF) is a progressive and fatal interstitial lung disease. The pathogenesis of interstitial lung diseases, including its most common form, IPF, remains poorly understood. Alveolar epithelial cell (AEC) apoptosis, proliferation, and accumulation of myofibroblasts and extracellular matrix deposition results in progressive loss of lung function in IPF. We found induction of tumor suppressor protein, p53, and apoptosis with suppression of urokinase-type plasminogen activator (UPA) and the uPA receptor in AECs from the lungs of IPF patients, and in mice with bleomycin, cigarette smoke, silica, or sepsis-induced lung injury. Treatment with the caveolin-1 scaffolding domain peptide (CSP) reversed these effects. Consistent with induction of p53, AECs from IPF lungs or mice with diverse types of lung injuries showed increased p53 acetylation and miR-34a expression with reduction in Sirt1. This was significantly reduced after treatment of wild-type mice with CSP, and UPA-deficient mice were unresponsive. Bleomycin failed to induce miR-34a in p53- or plasminogen activator inhibitor-1 (PAI-1)deficient mice. CSP-mediated inhibition of miR-34a restored Sirt1, suppressed p53 acetylation and apoptosis in injured AECs, and prevented pulmonary fibrosis (PF). AEC-specific suppression of miR-34a inhibited bleomycin-induced P53, PAI-1, and apoptosis and prevented PF, whereas overexpression of precursor-miR-34a increased p53, PAI-1, and apoptosis in AECs of mice unexposed to bleomycin. Our study validates $\mathrm{p53}-$ miR-34a feedback as a potential therapeutic target in PF. (Am J Pathol 2017, 187: 1016-1034; http://dx.doi.org/10.1016/j.ajpath.2016.12.020)
\end{abstract}

The tumor suppressor protein, p53, induces cell cycle arrest, apoptosis, senescence, and innate immunity. Induction of p53 occurs in response to DNA-damaging agents to protect against carcinogenesis. ${ }^{1-4}$ Distinct signaling mechanisms have evolved to induce p53 through posttranslational stabilization. Cell cycle arrest because of elaboration of p53 can be transient or permanent as in the case of senescence and promote alveolar epithelial cell (AEC) apoptosis, especially if the damage is severe and beyond repair. The outcomes are decided by the extent of p53 protein accumulation, with lower levels promoting cell cycle arrest and higher levels inducing apoptosis. The cellular responses of p53 are presumed to be mediated by its ability to activate or repress downstream target genes at the transcriptional or posttranscriptional levels. These include genes that express
Supported in part by Flight Attendant Medical Research Institute Clinical Innovator Awards 150063 and 082380 (S.S.), American Heart Association grant GRNT19020001 (S.S.), and NIH grants R01HL133067-01 and R21ES025815 (S.S.).

Disclosures: S.I. has an equity position as founder of Lung Therapeutics Incorporated (LTI) and serves as an unpaid member of the Board of Directors and Chief Scientific Officer. Although the company is commercializing single-chain urokinase for the treatment of empyema and complicated parapneumonic pleural effusions, the present work is unrelated to that effort in any way. S.S. and S.I. are inventors of a caveolin scaffolding domain peptide fragment with the rights licensed to LTI but the present work does not include the use of that intervention and otherwise is unrelated to commercialization of that peptide.

Current address of S.K.S., Department of Biochemistry, University of Iowa Carver College of Medicine, Iowa City, IA. 
proteins or noncoding RNAs responsible for cell cycle arrest, proliferation, cell signaling, senescence, and apoptosis. ${ }^{5-9}$

Lung injury due to exposure to DNA-damaging agents, such as bleomycin (BLM), cigarette smoke (CS), silica, or radiation, rapidly induces p53 expression in AECs. ${ }^{10-15}$ Likewise, hypoxia and hyperoxia augment p53 expression in AECs and other lung resident cells. ${ }^{16-18}$ Marked induction of p53 leads to apoptosis in AECs and subsequent activation and overgrowth of activated fibroblasts, in turn promoting fibrogenesis and the development of pulmonary fibrosis (PF). ${ }^{13,19}$ Consistent with these observations, AECs surrounding the fibrotic foci in the lungs of patients with idiopathic pulmonary fibrosis (IPF) exhibit increased apoptosis as well as increased p53 and plasminogen activator inhibitor-1 (PAI-1) expression. ${ }^{20-22}$ Similarly, large numbers of apoptotic AECs are detected in the lungs of mice with well-established PF long after (14 or 21 days) inception of BLM-induced fibrosing lung injury. ${ }^{12}$ These observations suggest that AECs continuously die in fibrotic lungs and are replaced by activated myofibroblasts or fibrotic lung fibroblasts, leading to destruction of lung architecture and progressive loss of lung function. AECs of patients with chronic obstructive pulmonary disease, emphysema, acute lung injury, acute respiratory distress syndrome, and diffuse alveolar damage characteristically exhibit increased AEC apoptosis and p53 expression, ${ }^{10-13}$ indicating a causal link between increased p53 and AEC apoptosis in diverse forms of lung diseases. Recent reports also reveal that emphysema and PF concurrently occur in approximately $10 \%$ of smokers. ${ }^{23,24}$ Similarly, patients who develop acute respiratory distress syndrome are more prone to the development of accelerated PF, which may likewise occur to some degree in up to $40 \%$ of patients. ${ }^{25,26}$ Increased numbers of apoptotic AECs in the lungs of patients with $\operatorname{IPF}^{19,22,27}$ or mice with established $\mathrm{PF}^{12}$ and protection against development of $\mathrm{PF}$ after transplantation of healthy AECs in mice with BLM $^{28}$ or silica-induced ${ }^{29}$ lung injury underlines the importance of AEC apoptosis in fibrogenic remodeling. Furthermore, resistance to BLM or silica-induced AEC apoptosis and PF because of deficiency in $\mathrm{p} 53$ or PAI-1 expression ${ }^{12,14}$ or inhibition of BLMinduced p53 from binding to endogenous urokinase-type plasminogen activator (uPA), uPA receptor (uPAR), and PAI-1 mRNAs ${ }^{13}$ demonstrates the contribution of increased p53 expression in AECs to the pathogenesis of lung injury and PF.

Besides transcriptional activation and repression control of protein-coding genes, p53 also regulates miRNA
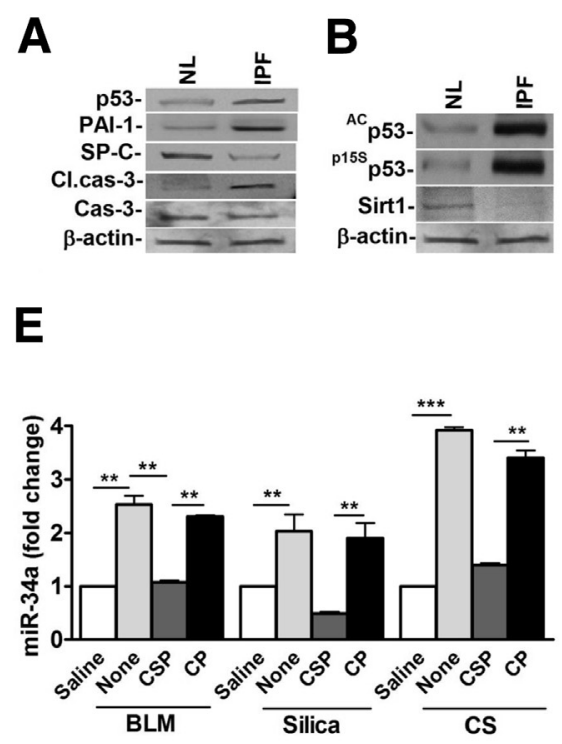

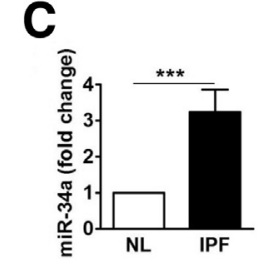

$\mathbf{F}$
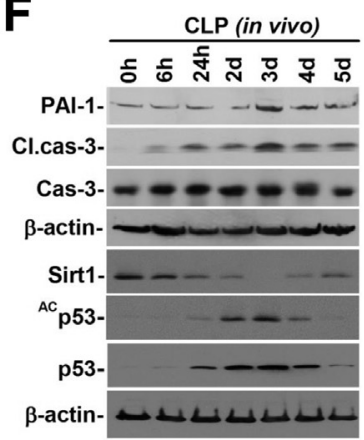
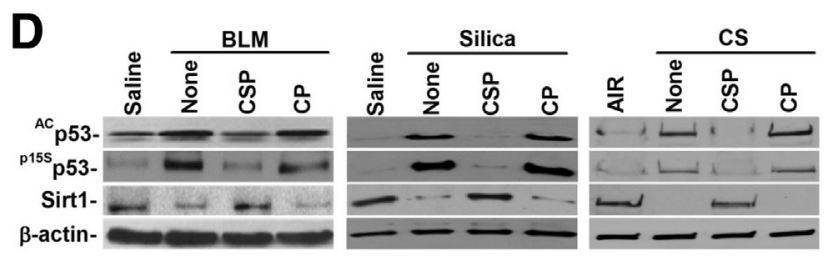

G

H
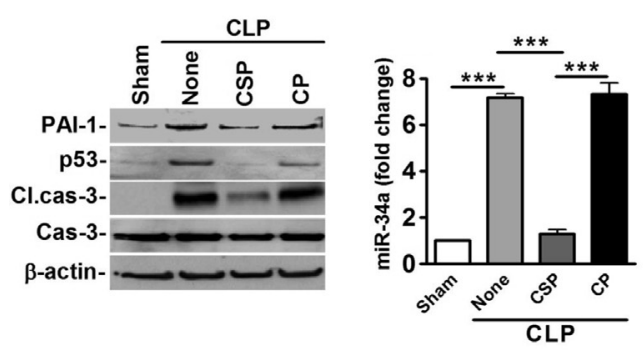

Figure 1 Caveolin scaffolding domain peptide (CSP) reverses bleomycin (BLM)-induced ${ }^{A c} p 53$ and miR-34a, and induces Sirt1 expression. A: Alveolar epithelial cells (AECS) isolated from the healthy donors (NL) and patients with idiopathic pulmonary fibrosis (IPF) were immunoblotted for changes in p53, PAI-1,

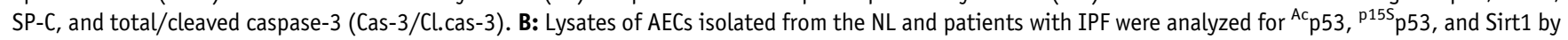
Western blotting. C: RNA from AECs of NL and IPF lungs was tested for changes in miR-34a by quantitative real-time RT-PCR. The bars represent the relative increase in miR-34a by AECs of IPF lungs compared to those obtained from NL controls after normalization with corresponding level of snRNA U6 in each sample. D: WT mice exposed to BLM or silica were i.p. injected with CSP, CP, or vehicle (none) 24 hours after injury. WT mice were exposed to air, or CS with or without CSP or CP for 20 weeks as described in Materials and Methods. AECs isolated from these mice 3 days later were immunoblotted for ${ }^{A C} p 53,{ }^{p 15 S}$ p53, and Sirt1. E: RNA from AECs of mice exposed to BLM, silica, or CS with or without CSP or CP was analyzed for miR-34a by quantitative real-time RT-PCR, as described in C. The bars represent the relative changes in miR-34a under different treatment conditions compared to saline controls from three experiments. F: AECs isolated from the lungs of WT mice 0 to 5 days after CLP injury were immunoblotted for PAI-1, Cl./total cas-3, Sirt1, ${ }^{A c}$ p53, p53, and $\beta$-actin. G: WT mice with CLP injury were i.p. injected with vehicle, CSP, or CP 24 hours after surgery. Control mice were sham operated. AECs from sham-operated or CLP mice treated with or without CSP or CP were isolated 3 days after sham operation or CLP. AEC lysates were immunoblotted for PAI-1, p53, Cl/total cas-3, and $\beta$-actin. H: RNA from AECs of mice subjected to sham operation or CLP as in G was analyzed for miR-34a by quantitative real-time RT-PCR. The bar represents relative changes in miR-34a after normalization for snRNA U6 under various conditions. Data are expressed as means \pm SD. $n=4$ (B and C). ${ }^{* *} P<0.01,{ }^{* *} P<0.001$. 
A

\begin{tabular}{|l|l|l|l|l|l|}
\hline CMV & $R$ & U5 \\
\hline
\end{tabular}

B

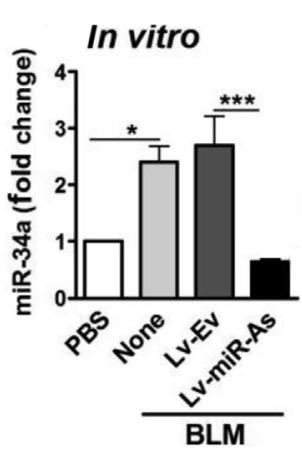

G

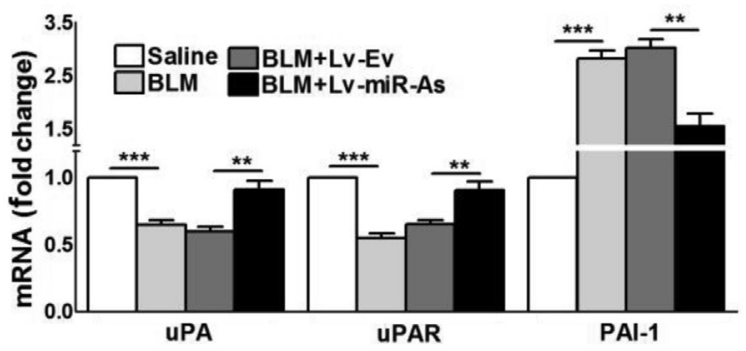

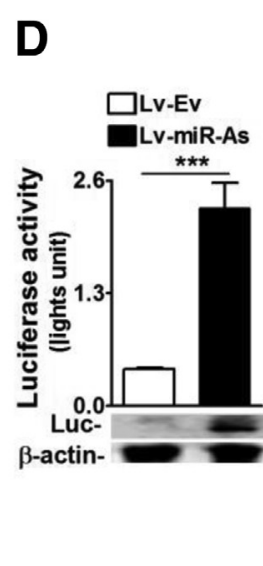

E

F
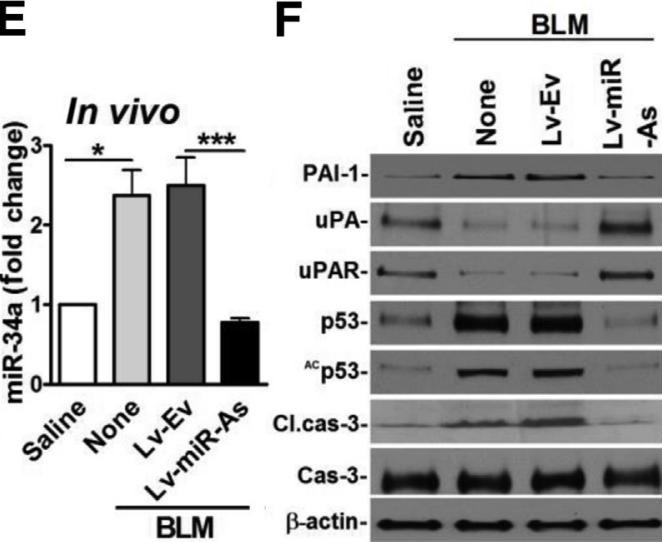

PAI-1-

UPA-

UPAR-

p53-

${ }^{\text {Ac }}$ p53-

Cl.cas-3-

Cas-3-

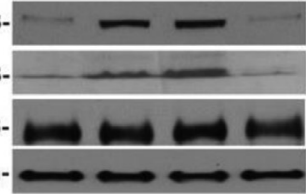

H
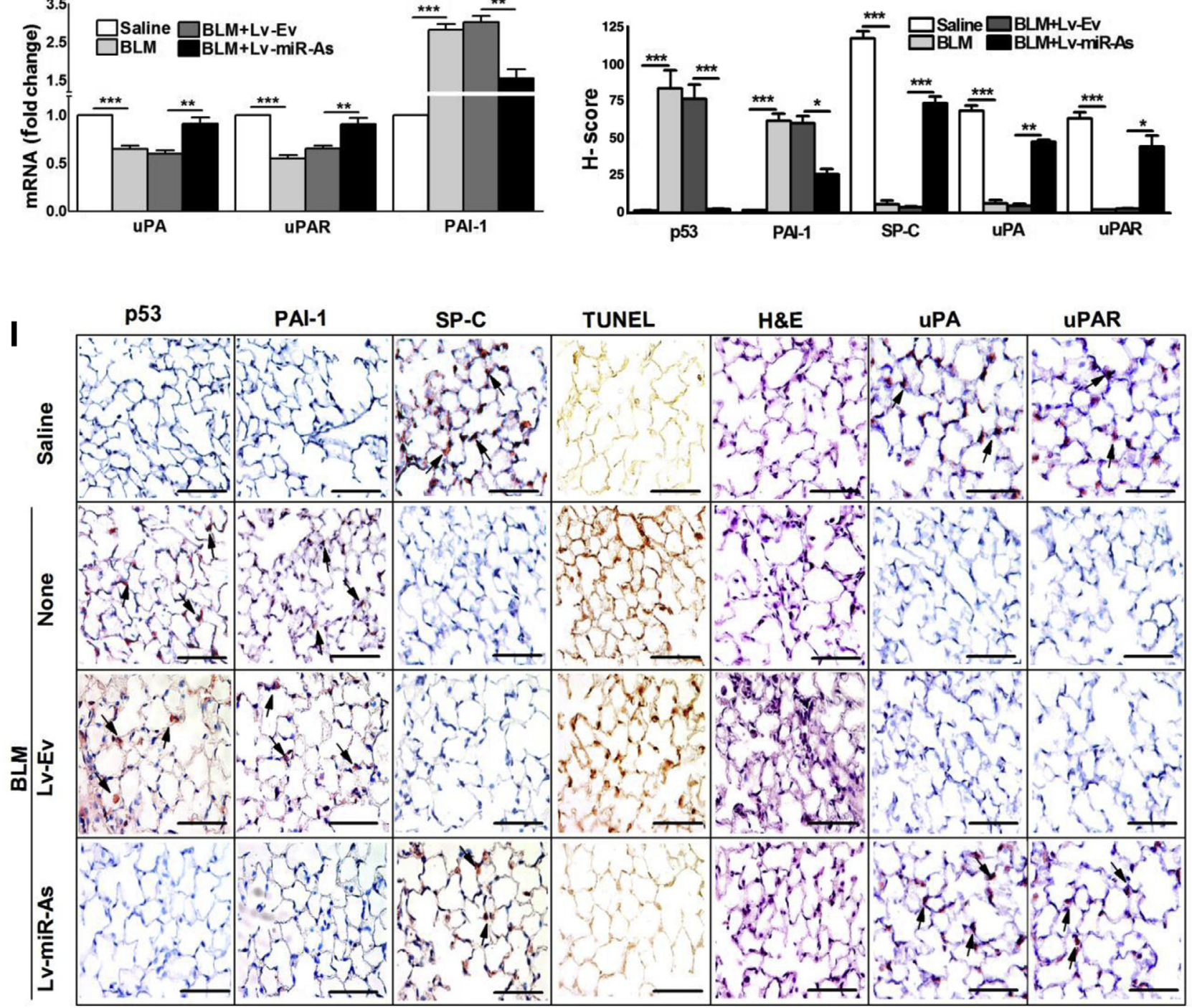
encoding genes. ${ }^{30,31}$ miRNAs are 18 to 25 nucleotide long noncoding RNAs, which regulate pathophysiological functions via expression of target genes at the posttranscriptional level. The process involves degradation, destabilization, ${ }^{32}$ or repression of translation, ${ }^{33}$ depending on the degree of homology of the miRNA with the target mRNA. Despite the fact that miRNAs are evolutionarily conserved, a single miRNA can target thousands of mRNAs, resulting in a lack of clarity about their true function or place in pathophysiologic events, including those that are central to the development of PF. Among these miRNAs, miR-34a appears to show marked induction by $\mathrm{p} 53 .{ }^{34} \mathrm{We}$ inferred that miR-34a may promote p53-mediated apoptosis, senescence, cell cycle arrest, and inhibition of proliferation. ${ }^{35}$ Interestingly, miR-34a inhibits deacetylation of p53 by blocking Sirt1 expression by binding to sequences present in the $3^{\prime}$ untranslated region of Sirt1 mRNA leading to increased acetylation of $\mathrm{p} 53 .^{36}$ These observations suggest that miR$34 \mathrm{a}-$ mediated acetylation of p53 could be a key effector of AEC apoptosis and development of PF through increased expression of p53 and by p53-mediated downstream changes in uPA fibrinolytic system cross talk. Currently available treatments are unable to target these interactions and prevent fibrogenesis. Therefore, we elucidated how $\mathrm{p} 53$ and miR-34a expression regulates AEC apoptosis and the development of $\mathrm{PF}$ through interaction with the major components of the uPA fibrinolytic system. Using isolated AECs from mice with BLM, silica, CS, and sepsis-induced lung injury, and a range of genetically altered mice subjected to BLM-induced lung injury, we further show how targeting this pathway mitigates AEC apoptosis and prevents PF.

\section{Materials and Methods}

Mice

Wild-type (WT) and p53- and PAI-1-deficient mice of C57BL-6 background were either purchased from The
Jackson Laboratory (Bar Harbor, ME) or bred at the University of Texas Health Science Center at Tyler. Breeding pairs of transgenic mice carrying SP-C ${ }^{\mathrm{Cre}}$ and $\mathrm{mTmG}$ genes ${ }^{(S P-C}{ }^{\text {Cre/mTmG }}$ mice) ${ }^{37}$ were kindly provided by Dr. Hal Chapman (University of California, San Francisco, CA). Transgenic uPA-deficient, or homozygous miR-34 $\mathrm{a}^{\mathrm{f} / \mathrm{fl}}$ and

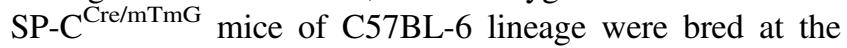
University of Texas Health Science Center at Tyler. All animal experiments were approved by the University of Texas Health Science Center at Tyler Institutional Animal Care and Use Committee.

\section{Isolation of AECs from Murine Lungs and Induction of BLM Injury in Vitro}

AECs were isolated from the lungs of mice and the purity of AEC preparations was confirmed by lithium carbonate staining and immunoblotting for surfactant proteins $\mathrm{A}, \mathrm{B}$, and C (SP-A, SP-B, and SP-C, respectively), as described previously $(90 \%$ to $95 \%) .{ }^{20}$ AECs were treated with $40 \mu \mathrm{g} / \mathrm{mL}$ BLM in the presence or absence of $10 \mu \mathrm{mol} / \mathrm{L}$ of caveolin-1 scaffolding domain peptide (CSP) or control scrambled peptide (CP) for 24 hours in AEC culture medium at $37^{\circ} \mathrm{C}$, as we described. ${ }^{20}$ The lysates and conditioned media were immunoblotted for changes in protein expression.

\section{Isolation of AECs from Control and IPF Lungs and Analysis of p53, PAI-1, and Apoptosis}

AECs were isolated from deidentified histologically normal lungs of control subjects and IPF donor lungs, as previously described. ${ }^{20,22}$ The purities of isolated cell preparations were confirmed, as described above, before immunoblotting. AEC lysates were subjected to Western blotting. In a separate experiment, human AECs purchased from ScienCell Res Lab (Carlsbad, CA) or isolated from normal lungs of control subjects were treated with BLM in the presence or absence of CSP or CP for 24 hours in AEC culture medium.

\footnotetext{
Figure 2 Inhibition of bleomycin (BLM)-induced miR-34a in alveolar epithelial cells (AECs) suppresses ${ }^{A c}$ p53, p53, PAI-1, and apoptosis, and restores uPA expression. A: Schematic diagrams show lentivirus vector (Lv) harboring SP-B promoter (SP-B) expressing luciferase gene (Luc) and miR-34a-As (miR-As). B: AECs isolated from WT mice were treated with BLM and exposed to Lv alone (Lv-Ev) or Lv with SP-B promoter containing luciferase gene plus miR-34a-As (Lv-miR-As). RNA isolated from these cells was analyzed for miR-34a by quantitative real-time RT-PCR. C: WT mice were i.v. injected with Lv-Ev or Lv-miR-As through their orbital plexus. Mice were exposed to intranasal BLM 24 hours after Lv transduction. Lung sections were tested for luciferase antigen expression by immunohistochemical (IHC) analyses. Arrows indicate positive staining. D: AECs isolated from WT mice were exposed to either Lv-Ev or Lv-miR-As and later treated with BLM as in C. The lysates of AECs isolated 3 days after BLM injury were tested for luciferase activity by chemiluminescent assay. The AEC lysates were also tested for luciferase and $\beta$-actin proteins by Western blotting (shown at bottom of the bars). E: RNA isolated from AECs of mice treated with saline, $B L M, B L M+L v-E v$, or BLM + Lv-miR-As were analyzed for changes in miR-34a by quantitative real-time RT-PCR. Experiments were repeated two times. F: AECs were isolated from WT mice treated as in D were immunoblotted for uPA, uPAR, PAI-1, ${ }^{\mathrm{Ac}} \mathrm{p53}, \mathrm{p} 53$, cleaved (Cl.)/total caspase-3, and $\beta$-actin. G: RNA isolated from AECs of WT mice exposed to saline, BLM, Lv-Ev + BLM, and Lv-miR-As + BLM as described in D above was analyzed for uPA, UPAR, and PAI-1 mRNA by quantitative real-time RT-PCR. The values were normalized against corresponding levels of $\beta$-actin mRNA in each sample. H: Morphometric quantification by $\mathrm{H}$-scoring of IHC analyses from I depicting changes is shown as bar graph. I: Lung sections from three mice treated as in $\mathbf{E}$ were subjected to IHC analyses for p53, PAI-1, UPA, UPAR, and SP-C antigens as well as TUNEL and H\&E staining. Arrows indicate positive staining. Representative images from 10 fields are presented. The in vivo analyses depicted in all of the panels were performed 3 days after BLM exposure. Data are expressed as means \pm SD. Experiments were repeated at least two times, and the representative results are presented in each panel. $n=4$ per group (D); $n=5(\mathbf{E}-\mathbf{G})$. ${ }^{*} P<0.05,{ }^{*} P<0.01$, and $* * * P<0.001$. Scale bars $=100 \mu \mathrm{m}\left(\mathbf{C}\right.$ and I). Original magnification, $\times 20(\mathbf{C}$ and I $)$. CMV, cytomegalovirus; cPPT, central polypurine tract; H $3^{\prime} \mathrm{LTR}$ P(A), hybrid 3'LTR poly (A); R, R sequence; U5, U5 region; WPRE, posttranscriptional regulatory elements of woodchuck hepatitis virus.
} 
A

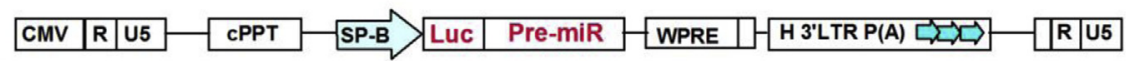

B

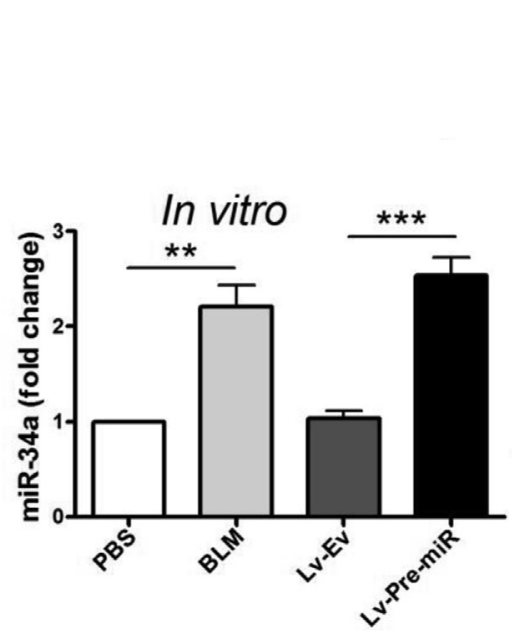

C

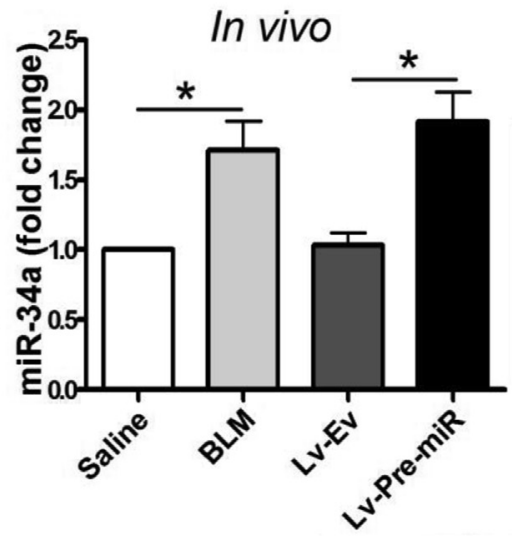

D

PAl-1-

UPA-

UPAR-

P53-

${ }^{A c}$ p53-

Cl.cas-3-

Cas-3-

$\beta$-actin-

E

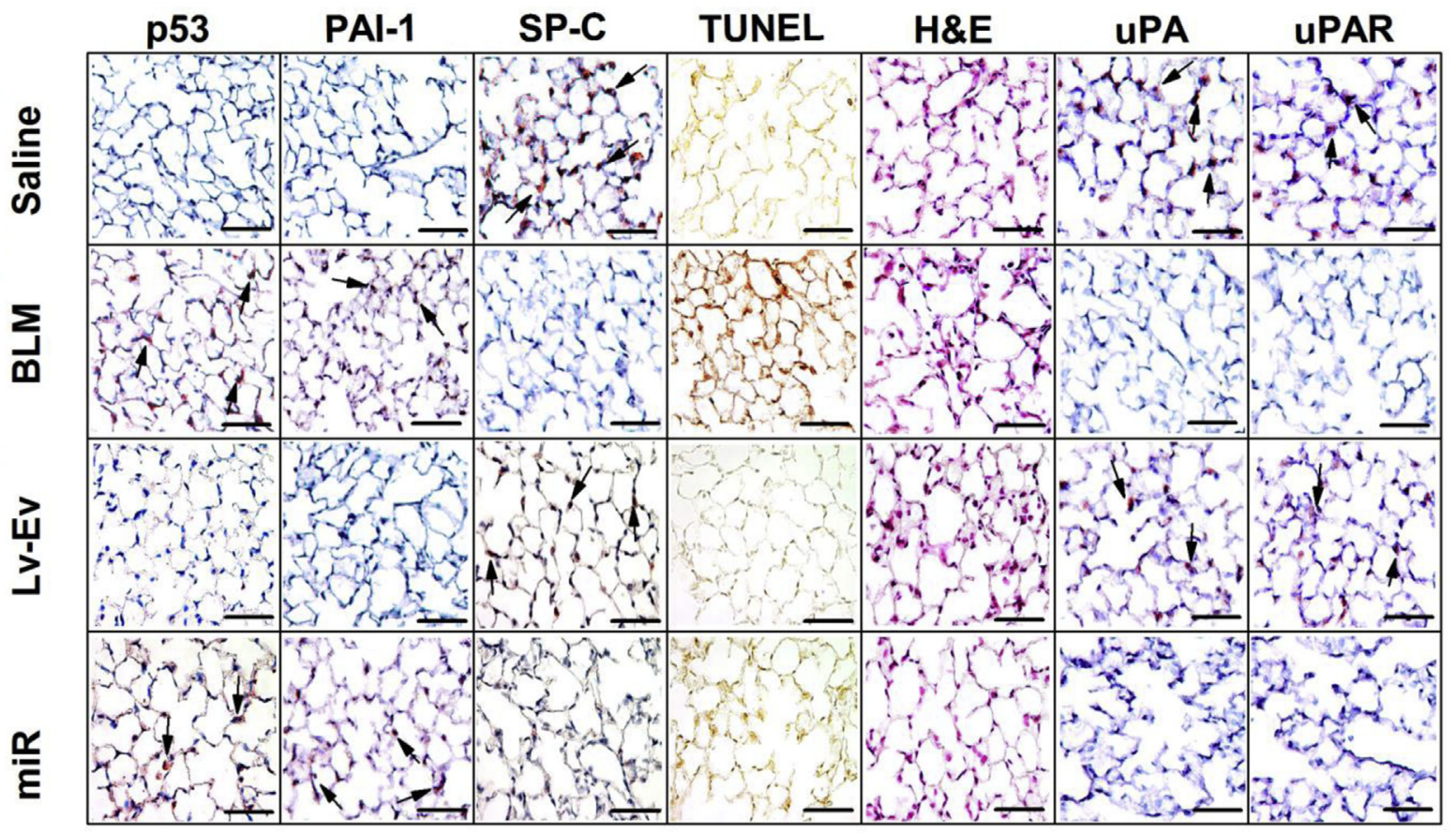

F

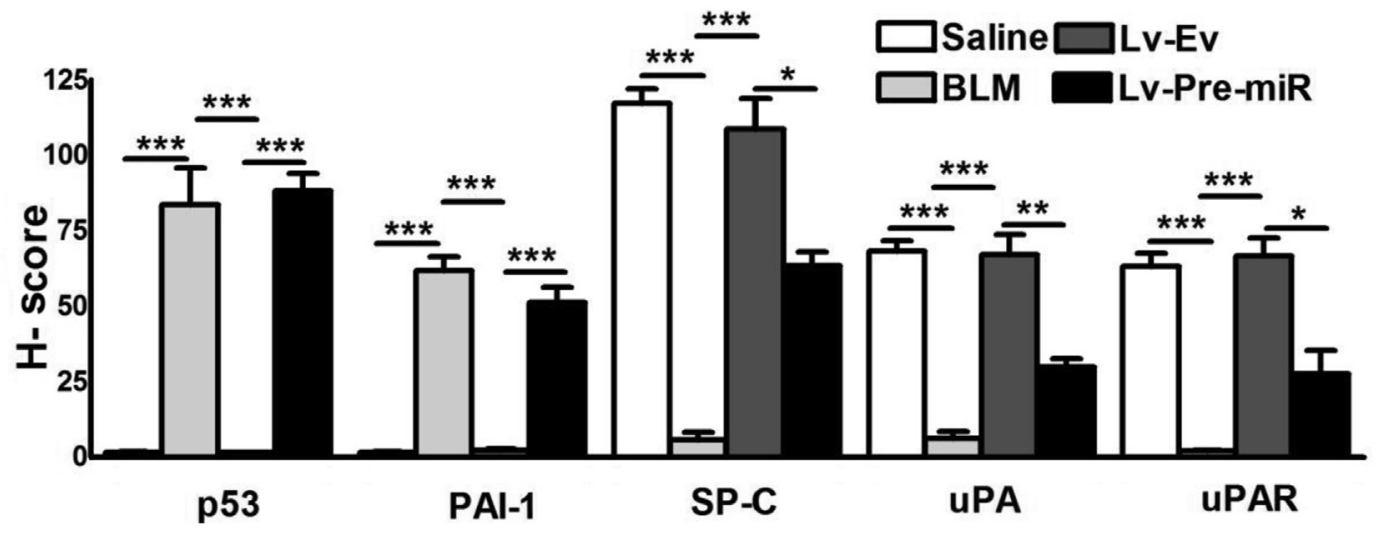


The lysates and conditioned media were subjected to Western blotting.

Induction of Mouse Models of BLM, Silica, CS, and Sepsis-Induced Lung Injury and Isolation of AECs

Mice were exposed to BLM or silica by intranasal instillation, as described earlier, ${ }^{12-14}$ whereas those exposed to saline were used as controls. In a separate experiment, WT mice were subjected to cecal ligation puncture (CLP) to induce septic lung injury and AECs isolated 0 to 5 days later, as described. ${ }^{38}$ In selected experiments, shamoperated mice were used as controls. Twenty-four hours after initiation of BLM, silica- or CLP-induced acute lung injury, mice were i.p. injected with either CSP or CP or remained untreated, as described. ${ }^{10,12,14}$ Three days later, mice were euthanized and AECs were isolated. For chronic lung injury, WT mice were exposed to passive CS from 40 research cigarettes over a 2-hour period 5 days/week for 5 days $\left(\leq 90 \mathrm{mg} / \mathrm{m}^{3}\right.$ total solid particulates) using a mechanical smoking chamber (Teague Enterprises, Davis, CA). Control mice were exposed to ambient air, as we reported elsewhere. ${ }^{10,11}$ After 4 weeks, mice exposed to CS were i.p. injected with CSP or CP once a week for 4 weeks and these mice were exposed to CS for a total of 20 weeks, as described earlier, ${ }^{11}$ then euthanized and AECs were isolated. The cell lysates and RNA isolated from AECs of WT mice with BLM, silica, CS, or CLP-induced lung injury and treated with or without CSP or CP, as well as AECs of control mice were subjected to Western blotting or real-time PCR to assess changes in ${ }^{\mathrm{p} 15 \mathrm{~S}} \mathrm{p} 53,{ }^{\mathrm{Ac}} \mathrm{p} 53$, p53, Sirt1, or cleaved and total caspase-3 proteins or miR-34a expression, respectively.

\section{Construction of Recombinant Lv Vectors}

cDNA corresponding to miR-34a antisense (miR-As) or Precursor-miR-34a (Pre-miR) sequences were subcloned into a pSMPUW universal promoterless lentivirus (Lv) vector purchased from Cell Biolab Inc. (San Diego, CA) downstream of the human SP-B 5'flanking DNA (-911/41) linked to the Luciferase reporter gene, as we described earlier. ${ }^{13} 293$ T cells were obtained from ATCC (Manassas,
VA) and maintained in RPMI 1640 media containing $10 \%$ heat-inactivated fetal bovine serum. The Lv vector constructs were transfected into 293T cells to obtain phage particles and viral titers, measured as per the manufacturer's protocol. Because SP-B promoter is AEC and Clara cell specific in the lungs, these constructs were later used to express the miR-As or Pre-miR in AECs in vivo.

\section{Expression of miR-As and Pre-miR-34a in Mouse Lung AECs in Vivo}

Six- to eight-week-old (with approximate $20 \mathrm{~g}$ body weight) WT mice or p53-, uPA-, and PAI-1-deficient mice were transduced with or without Lv vector harboring SP-B promoter alone ( $\mathrm{Lv}-\mathrm{Ev})$ or Lv having SP-B promoter expressing miR-As (Lv-miR-As) or Pre-miR (Lv-Pre-miR). Lvs were administered to anesthetized WT mice $(n=7)$ via the retro-orbital (i.v.) route, and 24 hours later BLM (40 $\mu$ g in $50 \mu \mathrm{L}$ saline) was administered via intranasal insufflation, as described. ${ }^{12,20}$ Mice were euthanized 3 days after BLM treatment, and the lungs were subjected to immunohistochemical (IHC) analysis or isolation of AECs to assess lung injury. Lung tissues of mice euthanized 21 days after BLM injury were evaluated for fibrotic changes, as described below.

\section{Inhibition of miR-34a Expression in AECs in Vivo}

To inhibit miR-34a expression, miR-34a floxed (miR-34a $\mathrm{a}^{\mathrm{f} / \mathrm{f}}$ ) mice with approximate $20 \mathrm{~g}$ body weight were i.v. administered with $30 \mu \mathrm{L}$ of $\mathrm{Lv}$ expressing Cre recombinase ( $\mathrm{Lv}-\mathrm{Cre}$ ). miR-34a $\mathrm{a}^{\mathrm{f} / \mathrm{fl}}$ mice exposed to Lv-Ev were used as controls. The mice were exposed to BLM 24 hours later. The mice were evaluated for apoptosis, and p53 and uPA system cross talk in AECs 3 days after intranasal exposure of BLM, and lung fibrosis 21 days later.

For generation of miR-34a conditional knockout $\left(\mathrm{miR}-34 \mathrm{a}^{\mathrm{cKO}}\right)$ mice lacking expression in AECs, miR-34a $\mathrm{a}^{\mathrm{f} / \mathrm{fl}}$ mice were cross-bred with SP-C Cre/mTmG mice. Firstgeneration heterozygous miR-34a ${ }^{\mathrm{fl}}$ and SP-C $\mathrm{C}^{\mathrm{Cre} / \mathrm{mTmG}}$ were backcrossed with homozygous miR-34 $\mathrm{a}^{\mathrm{f} / \mathrm{fl}}$ mice. The pups carrying only mutant miR-34a and SP-C ${ }^{\text {Cre/mTmG }}$ alleles were consecutively given i.p. injection of tamoxifen $(5 \mathrm{mg}$

\footnotetext{
Figure 3 Induction of ${ }^{A c} \mathrm{p} 53, \mathrm{p} 53$, and PAI-1 expression, and apoptosis by overexpression of miR-34a in alveolar epithelial cells (AECs). A: Schematic diagrams show Lv harboring SP-B promoter (SP-B) expressing luciferase gene (LuC) and precursor miR-34a (Pre-miR). B: AECS isolated from WT mice were transduced with either empty Lv (Lv-Ev) or Lv-Pre-miR in vitro. AECs treated with phosphate-buffered saline (PBS) or bleomycin (BLM) alone were used as controls for comparison. RNA isolated from AECs was analyzed for changes in miR-34a by quantitative real-time RT-PCR. C: WT mice were exposed to saline or BLM by nose or i.v. injected with Lv-Ev alone, or Lv-Pre-miR through their orbital plexus. All mice were euthanized 3 days later and AECs were isolated from these mice. RNA from AECs was analyzed for changes in miR-34a by quantitative real-time RT-PCR. D: Lysates from AECs of WT mice treated with saline, BLM, Lv-Ev, or Lv-Pre-miR as described in C were immunoblotted for ${ }^{A C} \mathrm{p} 53$ and p53, uPA, uPAR, and PAI-1, cleaved (Cl.)/total caspase-3, and $\beta$-actin. E: Lung sections were subjected to TUNEL and H\&E staining, and immunohistochemical analyses for p53, PAI-1, UPA, UPAR, and SP-C. Arrows indicate positive staining. Lung sections of saline- and BLM-treated mice shown in Figure 2I were used as controls for comparison. F: Bar graph represents morphometric quantification by H-scoring. Data are expressed as means \pm SD. Experiments were repeated at least two times. $n=5$ (B); $n=6$ per group (C); $n=3$ (E). ${ }^{*} P<0.05,{ }^{*} P<0.01$, and ${ }^{* * *} P<0.001$. Scale bars $=100 \mu \mathrm{m}(\mathrm{E})$. Original magnification, $\times 20(\mathrm{E})$. CMV, cytomegalovirus; cPPT, central polypurine tract; $\mathrm{H}$ 3'LTR P(A), hybrid 3'LTR poly (A); R, R sequence; U5, U5 region; WPRE, posttranscriptional regulatory elements of woodchuck hepatitis virus.
} 
in $100 \mu \mathrm{L}$ ) for 5 days to inhibit miR-34a expression through activation of Cre recombinase in AECs. The miR-34a ${ }^{\text {cKO }}$ mice were later exposed to BLM to induce lung injury in vivo.

\section{Preparation of AEC Lysates and Western Blotting}

AECs were isolated from the lungs of mice treated with BLM 3 days after inception of BLM lung injury, as described elsewhere. ${ }^{20}$ AEC lysates were separated using SDS-PAGE and electroblotted onto a nitrocellulose membrane. After blocking with $1 \%$ Tris-buffered saline-Tween-20 buffer containing bovine serum albumin, membrane was incubated with primary antibodies at $1: 1000$ dilutions at $4^{\circ} \mathrm{C}$ overnight, followed by reaction with donkey anti-rabbit horseradish peroxidase-conjugated secondary antibody at 1:3000 dilution for 1 hour at room temperature. Protein bands were visualized using the enhanced chemiluminescence detection method.

\section{Isolation and Analysis of RNA}

RNA extracted from isolated AECs or whole lung homogenates was subjected to RNase protection assay, as described earlier. ${ }^{39,40}$ In brief, isolated RNA was hybridized with ${ }^{32} \mathrm{P}$-labeled miR-34a and U6 antisense probes, and RNase-resistant ${ }^{32} \mathrm{P}$-labeled hybridization product was separated on a urea/polyacrylamide gel and subjected to autoradiography. The densities of individual bands were quantitated after normalization with the corresponding densities of U6 RNA in each sample. Total RNA extracted from isolated AECs or lung homogenates was also reverse transcribed and subjected to quantitative real-time RT-PCR for miR-34a, collagen-I (Col-1), fibronectin (FN), $\alpha$-smooth muscle actin ( $\alpha$-SMA), and tenascin-C (TN-C) transcripts.

\section{Hydroxyproline and Sircol Assays to Determine Lung Fibrosis}

Hydroxyproline contents of whole mouse lung tissues were determined according to a previously described method 21 days after initiation of BLM-induced lung injury. ${ }^{22}$ Lung homogenates were hydrolyzed in $6 \mathrm{~N} \mathrm{HCl}$ at $120^{\circ} \mathrm{C}$ for 24 hours. Hydrolysates were mixed with an equal volume of citrate/acetate buffer ( $\mathrm{pH}$ 6.0) and $100 \mu \mathrm{L}$ chloramine-T solution in a 96-well plate. The mixtures were incubated for 20 minutes at room temperature; then, $100 \mu \mathrm{L}$ Ehrlich's solution was added, and incubation was continued at $65^{\circ} \mathrm{C}$ for 18 minutes. Absorbance at $550 \mathrm{~nm}$ was measured, and hydroxyproline content in each lung tissue homogenate was determined from a standard curve. Collagen content in the lung tissues was also independently measured using a Sircol Collagen Assay Kit (Biocolor Ltd, Carrickfergus, UK). The results compared changes in Col- $1, \mathrm{FN}, \mathrm{TN}-\mathrm{C}$, and $\alpha$-SMA proteins in the lung homogenates by Western blotting.
IHC, H\&E, and Trichrome Staining of Lung Sections

Lungs extracted from mice 3 or 21 days after BLM exposure, as described above, were fixed under inflation via intratracheal instillation of Excell Plus solution (American MasterTech Scientific, Inc., Lodi, CA) at a constant pressure of $20 \mathrm{~cm} \mathrm{H}_{2} \mathrm{O}$ overnight. The lung sections (5 $\mu \mathrm{m}$ thick) of mice with or without BLM-induced injury or from patients with IPF or control donors were deparaffinized using xylene, followed by rehydration by incubation with $100 \%$ and $95 \%$ alcohol. Sections were then subjected to antigen retrieval via incubation in $10 \mathrm{mmol} / \mathrm{L}$ sodium citrate buffer $\left(\mathrm{pH} 6.0\right.$ ) at $95^{\circ} \mathrm{C}$ for 30 minutes, and were incubated with hydrogen peroxide for 30 minutes to quench endogenous peroxidase. Sections were incubated overnight with antibodies to PAI-1, p53, and cleaved caspase-3 purchased from Abcam (Cambridge, MA), Santa Cruz Biotechnology, Inc. (Dallas, TX), or Cell Signaling (Danvers, MA) or rabbit $\mathrm{IgG}$, which served as negative control, in phosphatebuffered saline containing $0.1 \%$ Tween-20, and processed for antigen detection (Lab Vision Corp., Fremont, CA). The deparaffinized lung sections were also subjected to terminal deoxynucleotidyl transferase-mediated dUTP nick-end labeling (TUNEL) and hematoxylin and eosin (H\&E) staining. Lung sections from mice euthanized 21 days after BLM injury or from patients with IPF or control donors were stained with H\&E and Masson's trichrome to detect interstitial accumulation of collagen and other matrix proteins. Morphometric analyses were performed by counting the positive cells in 10 randomly selected high-powered fields (magnification, $\times 20$ ) by $\mathrm{H}$-scoring per section, as we previously reported. ${ }^{10,41}$ The quantification of changes in the severity of lung fibrosis, quantitative fibrotic scale (Ashcroft scale and fibrotic score) was adopted to H\&E- and trichrome-stained sections, as described earlier. ${ }^{42,43}$

\section{Statistical Analysis}

Statistical significance between two groups was analyzed using $t$-test, and for multiple groups using two-way analysis of variance; $P<0.05$ was considered significant.

\section{Results}

Increased p53 expression and apoptosis in AECs of IPF lungs, and CSP inhibits p53 and PAI- 1 expression, and apoptosis in AECs of mice with lung injuries.

Lung sections of patients with IPF exhibited fibrotic foci, which are dense with collagen and other extracellular matrix proteins. ${ }^{22}$ To confirm increased expression of p53 and apoptosis in AECs, we isolated these cells from the IPF lungs and analyzed them for p53, PAI-1, and active caspase-3. Basal levels of p53 and PAI-1 were elevated in AECs from IPF lungs compared to AECs from control subjects (Figure 1A). These cells also showed activation of caspase-3, indicating increased AEC apoptosis. 

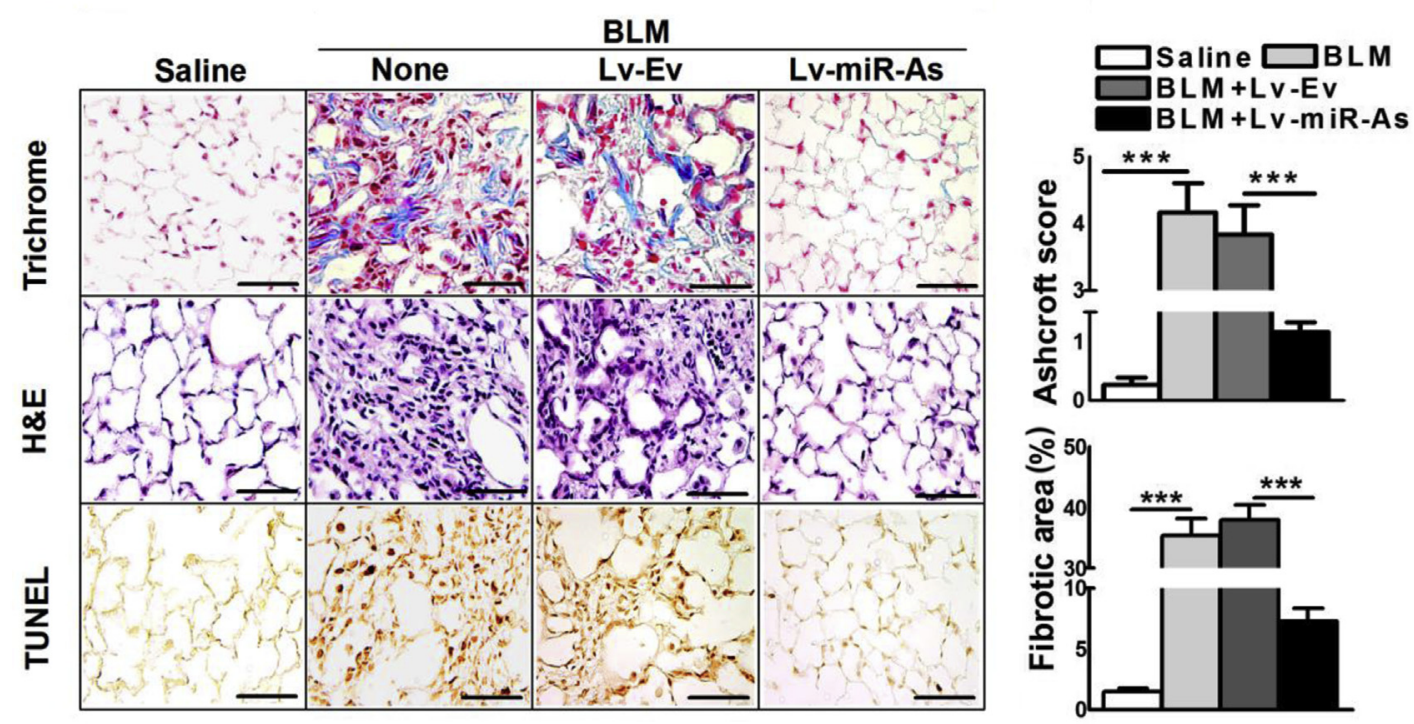

C
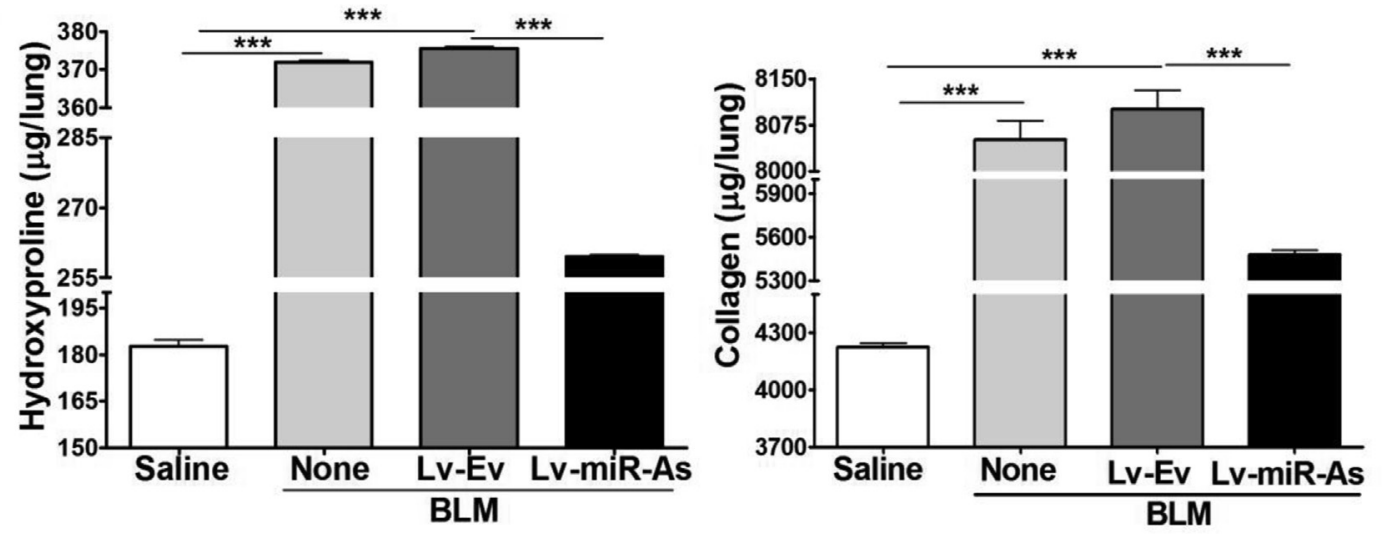

$\mathbf{E}$

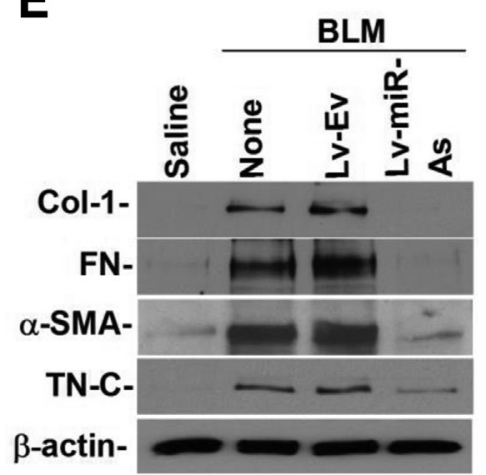

$\mathbf{F}$

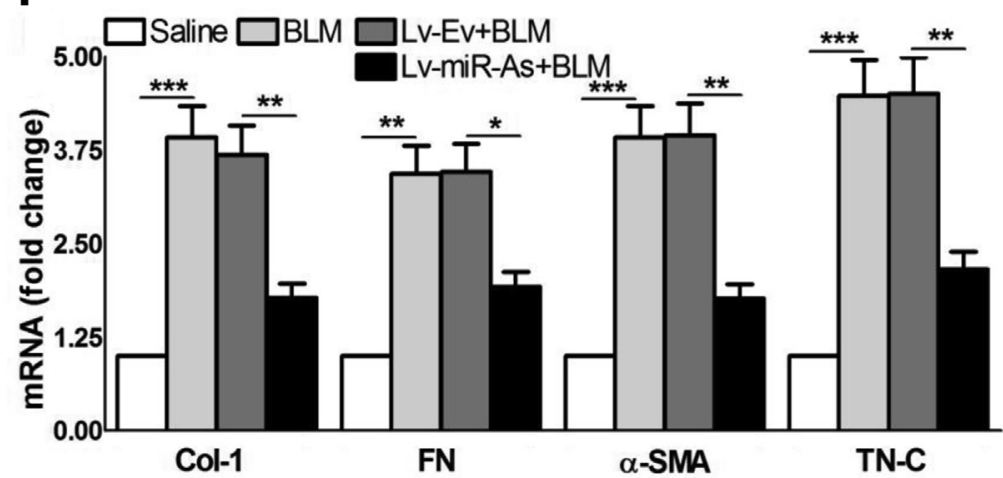

Figure 4 Inhibition of bleomycin (BLM)-induced miR-34a in alveolar epithelial cells (AECs) prevents development of lung fibrosis. A: WT mice were i.v. injected with Lv-Ev, or Lv-miR-As through their orbital plexus. Mice were exposed to intranasal BLM 24 hours after Lv transduction and euthanized 21 days after BLM injury. Lung sections from mice exposed to saline, BLM, Lv-Ev + BLM, and Lv-miR-As + BLM were subjected to trichrome, H\&E, and TUNEL staining. B: Ashcroft and fibrotic score of lung sections. Whole lung homogenates from mice euthanized 21 days after BLM injury were analyzed for hydroxyproline content (C) and collagen (D) by Sircol assay. E: Lung homogenates were immunoblotted for Col-1, $\alpha$-SMA, FN, or TN-C. F: RNA isolated from the lungs of mice euthanized 21 days after BLM injury was analyzed for Col-1, $\alpha$-SMA, FN, or TN-C mRNA by quantitative real-time RT-PCR. Each experiment was repeated at least two to three times. Each experiment in $\mathbf{C}$ and $\mathbf{D}$ was performed at least three times. Data are expressed as means \pm SD. $n=3$ mice per group (C and $\mathbf{D}$ ). ${ }^{*} P<0.05,{ }^{* *} P<0.01$, and ${ }^{* * *} P<0.001$. Scale bars $=100 \mu \mathrm{m}(\mathbf{A})$. Original magnification, $\times 20(\mathbf{A})$. 
A B C
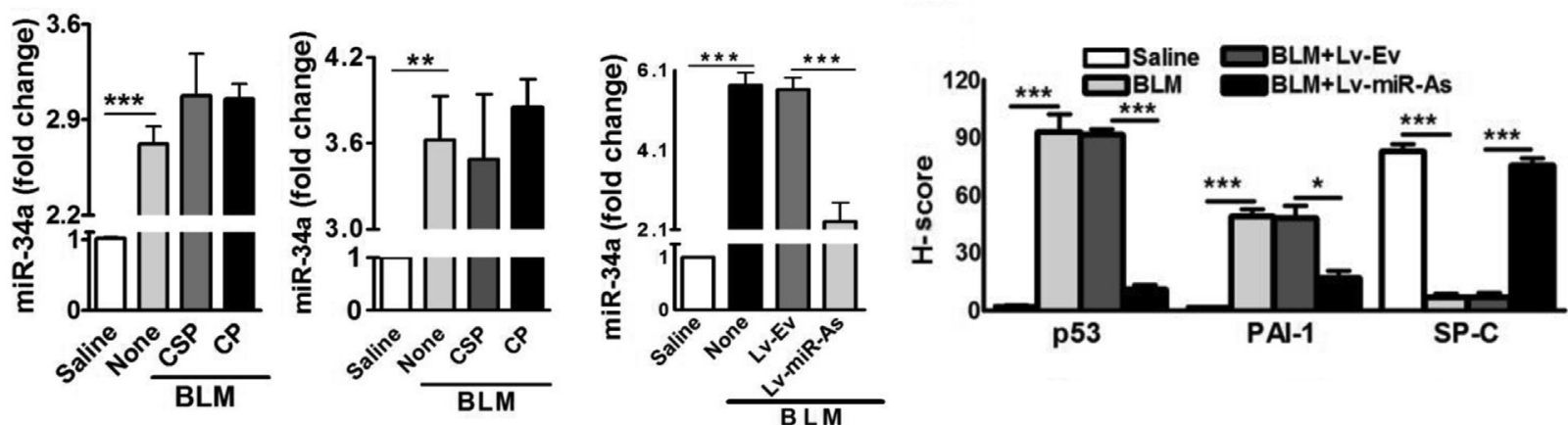

E

PAl-1

SP-C

H\&E

TUNNEL

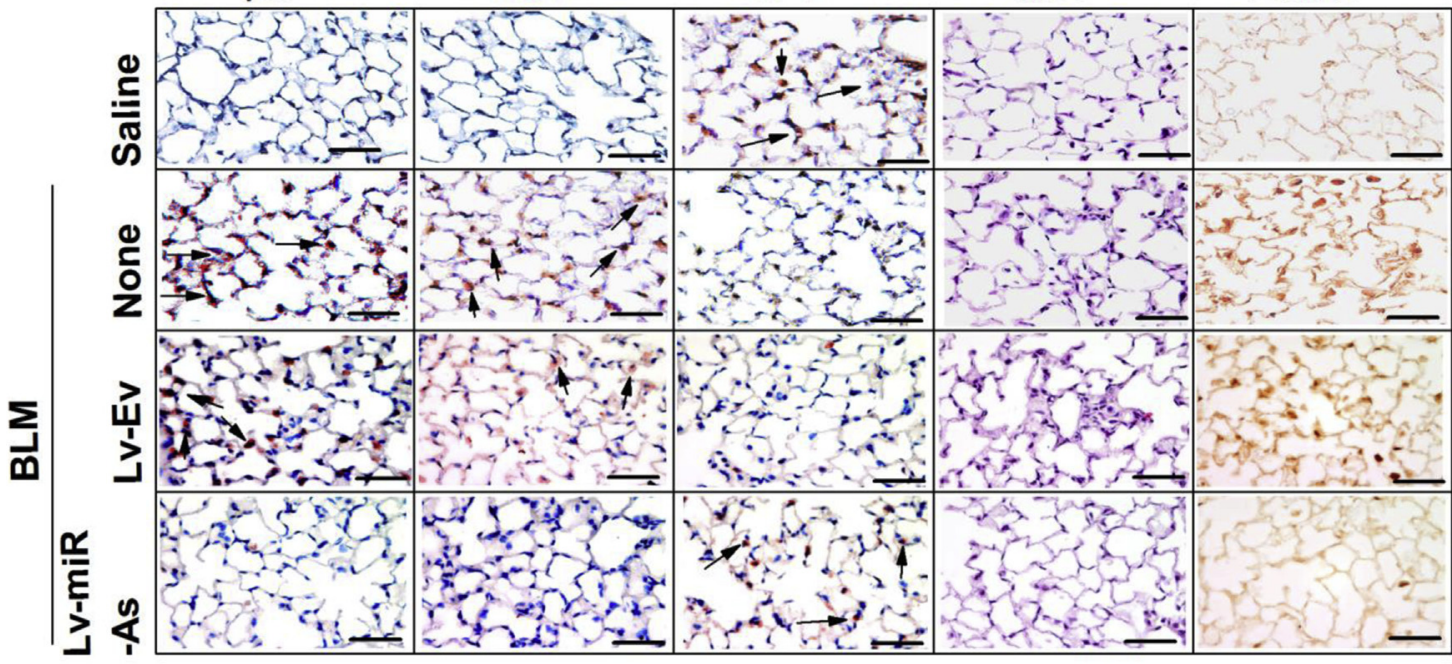

F

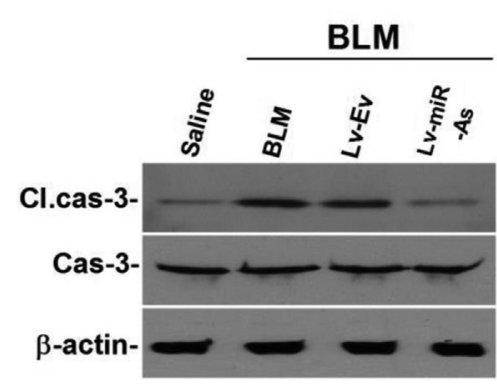

H

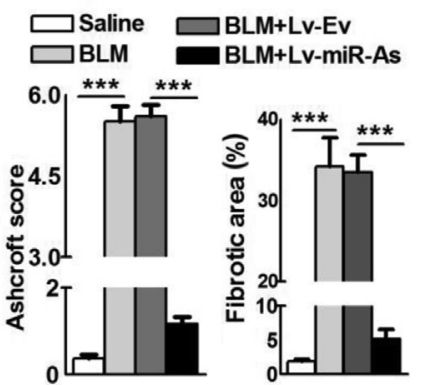

G
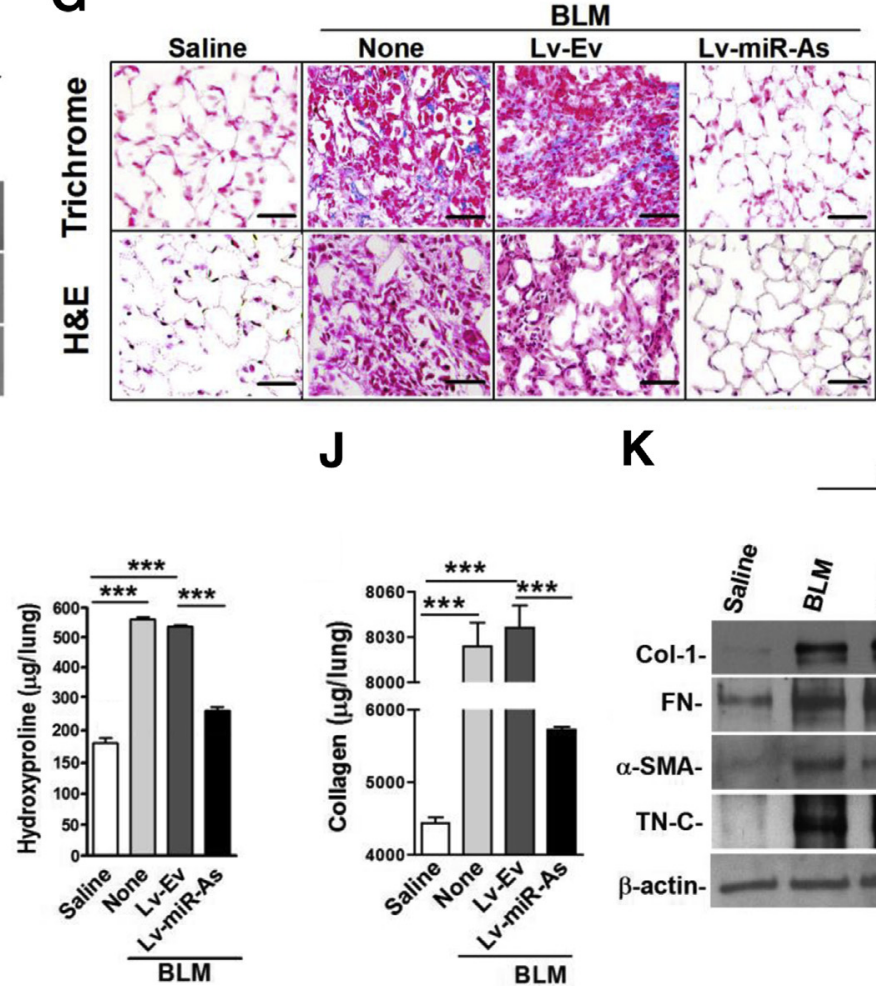

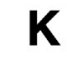

K
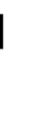
Immunofluorescence staining for p53, PAI-1, SP-C, and cleaved caspase- 3 and colocalization demonstrated that AECs surrounding fibrotic foci showed elevated staining for p53 antigens, active caspase-3, and PAI-1, indicating apoptosis of encircling AECs. Quantitation indicated that $74.1 \%$ of the PAI-1 antigen and $85.4 \%$ staining for p53 colocalized with SP-C-positive AECs; $66.6 \%$ of fluorescence for p53 antigen colocalized with AECs, exhibiting activated caspase-3. These findings collectively support a causal link between induction of p53 and PAI-1 and AEC apoptosis. Acetylation of p53 ( ${ }^{\mathrm{Ac}} \mathrm{p} 53$ ) apart from serine 15 phosphorylation of $\mathrm{p} 53\left({ }^{\mathrm{p} 15 \mathrm{~S}} \mathrm{p} 53\right)$ inhibits $\mathrm{p} 53$ interaction with $\mathrm{mdm} 2$, leading to increased stabilization and activation of $\mathrm{p} 53 .{ }^{44}$ In addition, the literature suggests that increased affinity of p300 to ${ }^{\mathrm{p} 15 \mathrm{~S}} \mathrm{p} 53$ proteins potentiates ${ }^{\mathrm{Ac}} \mathrm{p} 53 .{ }^{45} \mathrm{We}$ therefore tested AEC lysates for ${ }^{\mathrm{p} 15 \mathrm{~S}} \mathrm{p} 53$ and ${ }^{\mathrm{Ac}} \mathrm{p} 53$ and found that both posttranslational modifications of p53 were increased in AECs from IPF lungs (Figure 1B). Sirt1 expression was reduced in these cells. Because p53 induces miR-34a transcription, whereas the latter inhibits Sirt1 translation by binding to sequences in the p53 $3^{\prime}$ untranslated region, we next tested AECs for miR-34a expression. Consistent with increased ${ }^{\mathrm{p} 15 \mathrm{~S}} \mathrm{p} 53$ and ${ }^{\mathrm{Ac}} \mathrm{p} 53$, baseline miR34a was significantly increased in AECs from IPF lungs compared to AECs of control subjects (Figure 1C).

Consistent with changes in human AECs of IPF lungs, mice with $\mathrm{BLM}^{13}$ or silica ${ }^{14}$ or $\mathrm{CS}$-induced ${ }^{41}$ lung injury showed elevated levels of $\mathrm{p} 53$, and PAI-1 expression, and caspase- 3 activation, indicating AEC apoptosis. To confirm that the above findings applied to animal models, we next exposed mice to BLM or silica or chronic CS to induce lung injury. Next, AECs isolated from these mice were tested for changes in ${ }^{\mathrm{P} 15 \mathrm{~S}} \mathrm{p} 53,{ }^{\mathrm{Ac}} \mathrm{p} 53$, and Sirt1 expression. Consistent with induction of total p53, ${ }^{11,12,14}$ BLM or silica or chronic CS exposure induced lung injury in mice caused increased accumulation of ${ }^{\mathrm{p} 15 \mathrm{~S}} \mathrm{p} 53$ and ${ }^{\mathrm{Ac}} \mathrm{p} 53$ in AECs. These were markedly reduced in mice with BLM or silica or CS exposure injury that were treated with CSP, whereas ${ }^{\mathrm{p} 15 \mathrm{~S}} \mathrm{p} 53$ and ${ }^{\mathrm{Ac}} \mathrm{p} 53$ levels were still elevated in AECs of control mice exposed to $\mathrm{CP}$ (Figure 1D).

Because Sirt1 deacetylates p53 and its expression is reduced in AECs of IPF lungs, we determined if loss of
Sirt1 contributes to increased ${ }^{\mathrm{Ac}} \mathrm{p} 53$ in injured AECs. Interestingly, we found marked reduction in baseline expression of Sirt1 in AECs of mice after BLM-, silica-, or CS-induced injury, which was restored after treatment with CSP. This indicates that CSP-mediated suppression of p53 involves its deacetylation through restoration of Sirt1. As p53 induces miR-34a promoter transactivation, we next tested AECs for miR-34a expression by quantitative realtime RT-PCR (Figure 1E) and RNase protection assay (not shown). Consistent with the induction of p53, we also found twofold to fourfold increases in miR-34a expression in AECs after BLM, silica, or CS exposure lung injury. miR-34a expression was significantly reduced after treatment of BLM- or silica- or CS-injured mice with CSP (Figure 1E). AECs of control mice with BLM, silica, or CS lung injury and exposed to $\mathrm{CP}$ expressed elevated levels of miR-34a. In addition, we found that sepsis-induced lung injury in WT mice because of CLP caused a time-dependent increase in AEC p53 and PAI-1 expression, and activation of caspase-3, indicating apoptosis of AECs (Figure 1F). These changes were associated with induction of ${ }^{\mathrm{Ac}} \mathrm{p} 53$ and suppression of baseline Sirt1. Furthermore, treatment of WT mice 24 hours after CLP with CSP suppressed p53 and PAI-1 expression and apoptosis in AECs, which remained elevated in control mice exposed to $\mathrm{CP}$ (Figure 1G). Analysis of RNA isolated from AECs for miR34a revealed that CSP treatment also significantly reduced its expression in sepsis-induced lung injury (Figure $1 \mathrm{H}$ ). These findings indicate that miR-34a-mediated suppression of Sirt1 contributes to increased ${ }^{A c}$ p53 during acute and chronic lung injury, which can be reversed by targeting miR-34a in AECs.

\section{miR-34a Promotes AEC Apoptosis through p53-uPA System Cross Talk during BLM Injury}

We constructed an Lv vector harboring SP-B promoter plus luciferase and miR-As to inhibit miR-34a expression in AECs (Figure 2A). We next transduced AECs with Lv-miRAs in vitro and found significant reduction in BLM-induced miR-34a (Figure 2B). This reduction in BLM-induced miR34a occurred without suppression of luciferase expression

\footnotetext{
Figure 5 Caveolin scaffolding domain peptide (CSP) fails to inhibit bleomycin (BLM)-induced miR-34a expression in uPA-deficient mice. RNA from isolated alveolar epithelial cells (AECs; A) or lung homogenates of uPA-deficient mice exposed to saline, BLM, BLM + CSP, or BLM + CP (B) 3 days after BLM injury were analyzed for miR-34a by quantitative real-time RT-PCR. C: uPA-deficient mice were i.v. injected with Lv-Ev or Lv-miR-As through their orbital plexus. Later, mice were exposed to intranasal BLM 24 hours after Lv transduction. AECs were isolated from mice exposed to saline, BLM alone, BLM + Lv-Ev, and BLM + Lv-miR-As 3 days after BLM exposure. RNA from AECs was tested for miR-34a by quantitative real-time RT-PCR. D: Histopathological quantification of changes in p53, PAI-1, and SP-C by H-score of immunohistochemical (IHC) analyses from Figure 3E is depicted in bar graph. E: Lung sections from uPAdeficient mice as described above were subjected to TUNEL and H\&E staining or IHC analyses to evaluate changes in p53, PAI-1, and SP-C expression. Arrows indicate positive staining. F: AECs were isolated from mice treated with saline, BLM alone, BLM + Lv-Ev, and BLM + Lv-miR-As as described in C. The lysates were immunoblotted for cleaved ( $\mathrm{Cl}$.)/total caspase-3 and $\beta$-actin. G: Lung sections of uPA-deficient mice treated as described above and euthanized 21 days after BLM injury were subjected to trichrome and H\&E staining. H: Bars represent morphometric quantification by Ashcroft scoring. Lung homogenates from uPA-deficient mice euthanized 21 days after BLM injury were evaluated for PF by testing for hydroxyproline content (I) or collagen (J) by Sircol assay. K: Lung homogenates were immunoblotted for Col-1, FN, $\alpha$-SMA, or TN-C. Each experiment was repeated at least twice Eeach experiment in I and $\mathbf{J}$ was performed at least three times. Data are expressed as means \pm SD. $n=6(\mathbf{B}) ; n=5(\mathbf{C}) ; n=3$ mice per group (I and $\mathbf{J})$. ${ }^{*} P<0.05,{ }^{* *} P<0.01$, and ${ }^{* * *} P<0.001$. Scale bars $=100 \mu \mathrm{m}(\mathbf{E}$ and $\mathbf{G})$. Original magnification, $\times 20(\mathbf{E}$ and $\mathbf{G})$.
} 
by miR-34a-As (data not shown). Control AECs treated with $\mathrm{Lv}$ alone ( $\mathrm{Lv}-\mathrm{Ev}$ ) failed to suppress miR-34a. We later transduced BLM-injured mice with Lv-miR-As constructs and analyzed the lung sections for luciferase expression. Lung sections of mice exposed to BLM and transduced with Lv-miR-As showed increased staining for luciferase antigen with a transduction efficiency of approximately 90\% (Figure 2C). This is consistent with efficiency of Lv transduction reported earlier. ${ }^{13}$ Analyses of lysates from isolated murine AECs also revealed increased luciferase activity and antigen expression (Figure 2D). Control AECs of mice exposed to Lv-Ev showed little luciferase activity or antigen expression, indicating successful AEC-specific expression of chimeric luciferase-miR-34a-As cDNA in BLM-injured mice transduced with Lv-miR-As construct. We next sought to determine the expression of miR-34a in isolated AECs and found a significant decline of BLM-induced miR34a expression in mice transduced with $\mathrm{Lv}$-miR-As in vivo (Figure 2E). Consistent with increased miR-34a in AECs of WT mice after BLM injury (Figure 1E), Lv-Ev failed to suppress miR-34a in AECs of mice exposed to BLM.

Western blotting of AEC lysates of mice with BLM lung injury and exposed to Lv-miR-As showed marked suppression of ${ }^{A c}$ p53 and total p53, and PAI-1 (Figure 2F). These changes were associated with reduction in activation of caspase-3, indicating that inhibition of miR-34a expression mitigates AEC apoptosis because of BLM injury. Analysis of RNA also revealed that inhibition of miR-34a restored UPA and UPAR mRNA in AECs, while blocking induction of PAI-1 mRNA (Figure 2G). IHC analyses and $\mathrm{H}$-scoring of lung sections (Figure 2, H and I) showed significant suppression of $\mathrm{p} 53$ and PAI-1 compared to that in the lung tissues of mice exposed to BLM and transduced with Lv-Ev. These changes were associated with restoration of SP-C expression, indicating increased numbers of AECs. TUNEL staining confirmed less AEC apoptosis after inhibition of BLM-induced miR-34a. This is consistent with significant reduction of TUNEL-positive cells in lung section of p53-deficient mice exposed to BLM despite a delay in DNA repair. ${ }^{13,46} \mathrm{H} \& \mathrm{E}$ staining of lung sections also revealed that suppression of BLM-induced miR-34a expression in AECs reduced pulmonary inflammation consistent with reduced p53 and PAI-1 expression.

Inhibition of miR-34a using Lv-miR-As reduced BLMinduced AEC apoptosis, attenuated p53 expression, and reversed p53-mediated changes in UPA, uPAR, and PAI-1. To confirm that increased miR-34a-mediated induction of p53 contributes to AEC apoptosis through downstream changes in uPA and PAI-1, we constructed an Lv harboring SP-B promoter and Pre-miR (Lv-Pre-miR) (Figure 3A). Later, AECs were transduced with Lv-PremiR constructs in vitro. Analysis of total RNA from these cells revealed significant increases in miR-34a expression without BLM injury (Figure 3B). To test the effect of forced expression of Pre-miR in vivo, we transduced mice with Lv-Pre-miR and isolated AECs from these mice. Analysis of isolated AECs revealed increased miR34a expression in mice exposed to Lv-Pre-miR without BLM-induced lung injury in vivo (Figure 3C). Because miR-34a augments ${ }^{A c}$ p53, leading to its stabilization and increased steady-state expression, we next analyzed the AEC lysates for ${ }^{A c} \mathrm{p} 53$ and $\mathrm{p} 53$. Overexpression of miR$34 \mathrm{a}$ significantly increased both ${ }^{\mathrm{Ac}} \mathrm{p} 53$ and total p53 in AECs of mice without BLM lung injury (Figure 3D). We also found a parallel induction of PAI-1 and apoptosis, indicating that the process involves p53 and uPA system cross talk. IHC staining (Figure 3E) and H-scoring (Figure 3F) of lung tissues further confirmed elevated levels of TUNEL- and p53-positive AECs with reduced staining for SP-C in mice exposed to Lv-Pre-miR. This validates that miR-34a-mediated increments in p53 regulate AEC apoptosis.

\section{miR-34a Promotes BLM-Induced Lung Fibrosis through p53-uPA System Cross Talk}

AEC injury precedes development of $\mathrm{PF}^{12}$ and blockade of miR-34a expression attenuates AEC apoptosis after BLM injury (Figure 2). We therefore sought to test whether inhibition of BLM-induced miR-34a prevents development of PF. We euthanized the mice 21 days after BLM injury and analyzed the lung sections by Masson's trichrome and H\&E staining. We found minimal deposition of collagen and other matrix proteins in lung sections of mice transduced with LvmiR-As and exposed to BLM, whereas control mice treated with BLM alone or Lv-Ev and BLM showed intense staining (Figure 4A). Morphometric quantification by Ashcroft scoring (Figure 4B) revealed significant protection against BLM-induced fibrogenesis in mice exposed to Lv-miR-As. $\mathrm{H} \& \mathrm{E}$ staining also revealed preservation of lung architecture after blockade of BLM-induced miR-34a. Consistent with our earlier reports, ${ }^{12}$ TUNEL staining of lung sections revealed the presence of apoptotic AECs even 21 days after initiation of BLM-induced lung injury, which was markedly reduced in mice transduced with Lv-miR-As. The protection against development of PF was independently confirmed by demonstration of significantly less total hydroxyproline and collagen content in whole lung homogenates of BLM mice treated with Lv-miR-As (Figure 4, C and D). These findings were independently confirmed by resistance of mice exposed to miR-34a-As and BLM to induce Col-1, $\alpha$-SMA, FN, and TN-C protein (Figure 4E) or mRNA (Figure $4 \mathrm{~F}$ ) in the lungs.

CSP, a 20-amino acid peptide derived from the caveolin1 scaffolding domain, inhibits p53 expression and reverses p53-mediated downstream inhibition of uPA expression to protect AECs in WT mice with BLM injury. ${ }^{12}$ However, unlike WT mice with BLM injury, CSP failed to inhibit BLM-induced AEC apoptosis or prevent lung fibrosis in uPA-deficient mice. We therefore analyzed AECs of uPAdeficient mice treated with CSP after BLM injury for miR-34a expression. Unlike WT mice and consistent with lack of p53 inhibition by CSP in uPA-deficient mice, ${ }^{12}$ there 
A

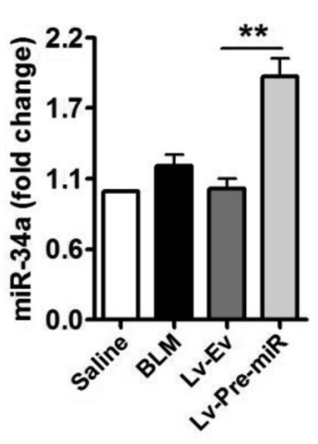

E

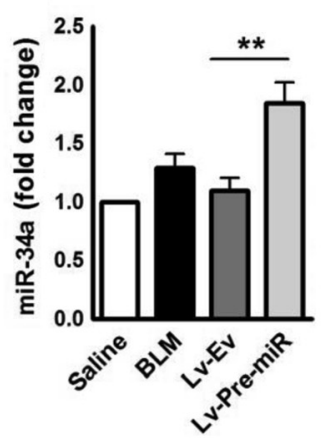

I

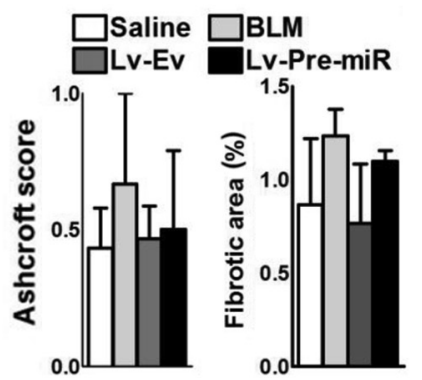

B

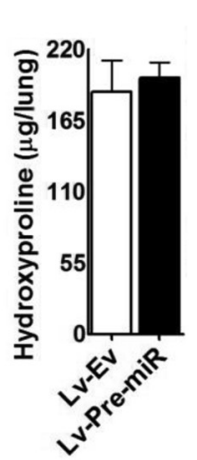

F

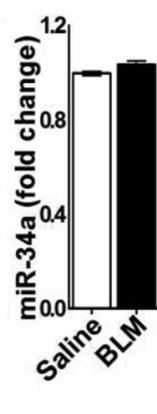

C

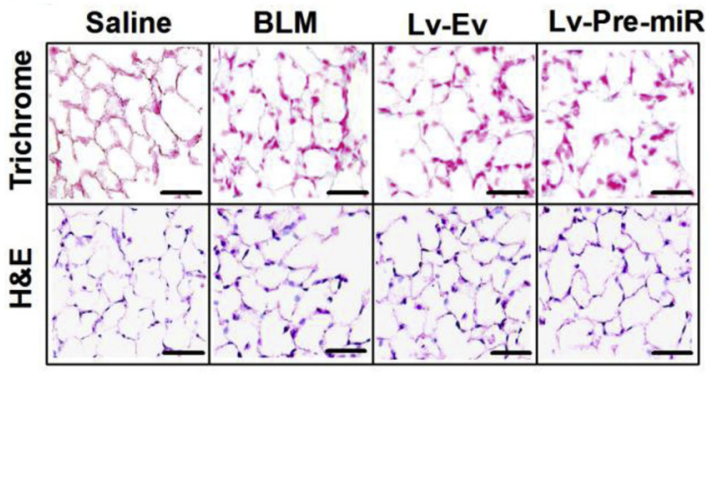

D

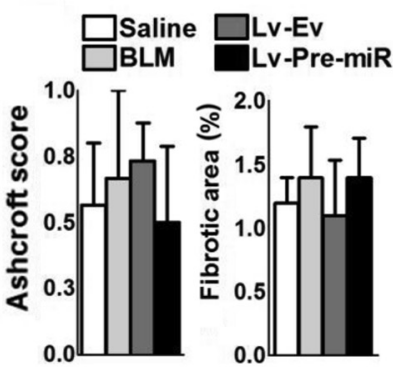

G

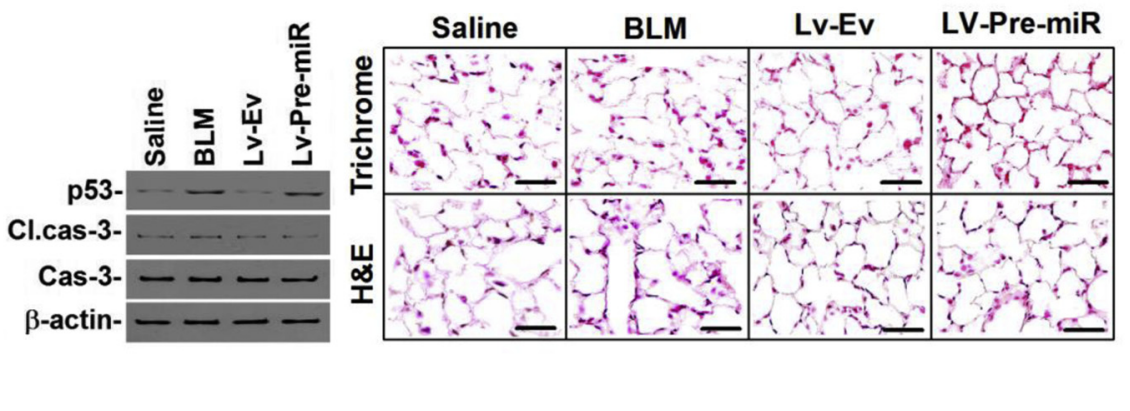

J

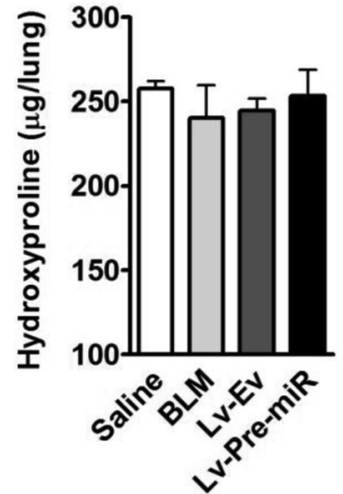

K

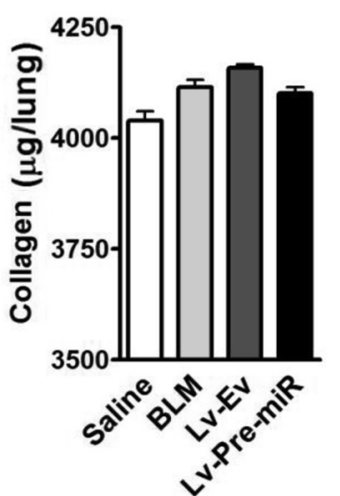

L

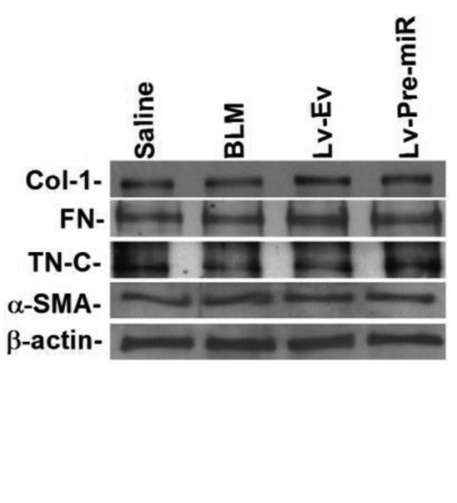

Figure 6 Role of p53 and PAI-1 in miR-34a-induced lung injury and development of lung fibrosis. A: p53-deficient mice exposed to saline or bleomycin (BLM) or i.v. injected with Lv-Ev alone, or Lv-Pre-miR through their orbital plexus. Alveolar epithelial cells (AECs) were isolated from these mice 3 days later and analyzed for changes in miR-34a by quantitative real-time RT-PCR. B: p53-deficient mice were i.v. injected with Lv-Ev or Lv-Pre-miR as described in A. The mice were euthanized 21 days later and whole lung homogenates were subjected to hydroxyproline assay to assess total collagen contents. C: p53-deficient mice exposed to saline, BLM, Lv-Ev, or Lv-Pre-miR were euthanized 21 days later. The lung sections were subjected to trichrome and H\&E staining. D: Bar graph represents morphometric quantitation of PF by Ashcroft and fibrotic scoring. E: PAI-1-deficient mice exposed to saline or BLM by intranasal instillation or i.v. injected with Lv-Ev, or Lv-Pre-miR through their orbital plexus as described in A. These mice were euthanized 3 days later. AECs were isolated from these mice and RNA obtained from AECs were analyzed for changes in miR-34a by quantitative real-time RT-PCR. F: PAI-1-deficient mice exposed to saline or BLM were euthanized 3 days after BLM injury. RNA obtained from the lung homogenate was tested for miR-34a by quantitative real-time RT-PCR. G: PAI-1-deficient mice exposed to saline, BLM, or i.v. injected with Lv-Ev alone, or Lv-Pre-miR as in $\mathbf{E}$ were euthanized 3 days later and isolated AECs from these mice. The AEC lysates were evaluated for p53, total/ cleaved (Cl.) caspase-3, and $\beta$-actin by Western blotting. H: PAI-1-deficient mice exposed to saline, BLM, Lv-Ev, or Lv-Pre-miR as described in $\mathbf{G}$ were euthanized after 21 days. Lung sections were subjected to trichrome and H\&E staining. I: Bars represent morphometric quantification of pathological changes by Ashcroft and fibrotic scoring. Lung homogenates of PAI-1-deficient mice exposed to saline, BLM, Lv-Ev, or Lv-Pre-miR and euthanized 21 days later as in $\mathbf{H}$ were tested for hydroxyproline content $(\mathbf{J})$ or collagen $(\mathbf{K})$ by Sircol assay. L: Lung homogenates from saline, BLM-, Lv-Ev-, or Lv-Pre-miR-treated PAI-1-deficient mice euthanized 21 days later were immunoblotted for Col-1, FN, $\alpha$-SMA, or TN-C. The experiments were repeated two to three times. Experiements in B were repeated at least two times. Experiments in $\mathbf{J}$ and $\mathbf{K}$ were performed at least three times. Data are expressed as means \pm SD. $n=6(\mathbf{A}$ and $\mathbf{E}) ; n=3$ mice per group $(\mathbf{B}, \mathbf{J}$, and $\mathbf{K}) ; n=8(\mathbf{H}) .{ }^{*} P<0.01$. Scale bar $=100 \mu \mathrm{m}(\mathbf{C}$ and $\mathbf{H})$. Original magnification, $\times 20(\mathbf{C}$ and $\mathbf{H})$. 
was no significant inhibition of miR-34a expression in either isolated AECs (Figure 5A) or the lungs (Figure 5B) of uPA-deficient mice exposed to BLM, BLM + CSP, or $\mathrm{BLM}+\mathrm{CP}$. We next expressed miR-As to directly suppress BLM-induced miR-34a expression in uPA-deficient mice. In contrast to CSP treatment, transduction of uPA-deficient mice exposed to BLM with miR-As significantly reduced miR-34a expression in AECs (Figure 5C). IHC analyses of lung sections showed suppression of p53 and PAI-1 expression and apoptosis, indicated by fewer TUNELpositive cells in the lung sections (Figure 5E). The lung sections of these mice also showed increased SP-C antigen staining, indicating preservation of AECs. This was further confirmed by histopathological quantification by $\mathrm{H}$-scoring (Figure 5D). Furthermore, analyses of lysates of isolated AECs showed reduction in BLM-induced apoptosis in mice exposed to Lv-miR-As (Figure 5F). Evaluation for lung fibrosis of trichrome and $\mathrm{H} \& \mathrm{E}$-stained lung sections (Figure 5G) and quantification by Ashcroft scoring (Figure 5H) 21 days after BLM lung injury proved suppression in collagen and other matrix protein deposits in the lungs of BLM + Lv-miR-As exposed mice versus those treated with BLM + Lv-Ev. Testing of hydroxyproline (Figure 5I) and collagen by Sircol assay (Figure 5J) in whole lung tissues also revealed significant reduction after inhibition of BLM-induced miR-34a expression. This was validated by measuring Col-1, $\alpha$-SMA, FN, and TN-C levels of the lungs by Western blotting, which showed significant reduction in BLM + miR-As treated mice (Figure 5K). Thus, our data support the novel concept that BLM-induced development of PF can be controlled by suppressing miR-34a expression and the process involves p53-mediated AEC apoptosis.

Because $\mathrm{p} 53$ promotes miR-34a transactivation, we tested whether BLM exposure induces miR-34a expression in $\mathrm{p} 53$ deficient mice. RNA isolated from the AECs or from the lung tissues (not shown) of p53-deficient mice treated with BLM failed to augment miR-34a expression 3 days after exposure (Figure 6A). These findings indicate that increased p53 expression is required for induction of miR-34a during BLM-lung injury. However, transduction of p53-deficient mice with Lv harboring SP-B promoter and Pre-miR-34a showed significant increase in miR-34a expression in AECs 3 days later. Analysis of lung homogenates 21 days after exposure of p53-deficient mice to Pre-miR-34a showed no increase in total hydroxyproline contents (Figure 6B), indicating resistance to development of lung fibrosis. Analysis of trichrome- or H\&E-stained lung sections (Figure 6C) and morphometric quantification by Ashcroft scoring (Figure 6D) showed lack of PF in p53-deficient mice with AEC-specific forced expression of Pre-miR-34a.

p53 also induces PAI-1 expression through increased mRNA synthesis ${ }^{47}$ and posttranscriptional stabilization of PAI-1 mRNA transcripts ${ }^{48}$; however, mice deficient in PAI1 expression resist BLM-induced AEC apoptosis and PF. Therefore, we next sought to assess whether BLM induces
miR-34a expression in PAI-1-deficient mice. Analysis of RNA from isolated AECs (Figure 6E) or of the lung tissues (Figure 6F) revealed that BLM exposure did not increase miR-34a. Because BLM failed to induce miR-34a in AECs of PAI-1-deficient mice, we next transduced these mice with Pre-miR-34a and analyzed AECs for miR-34a. Transduction of PAI-1-deficient mice with Pre-miR-34a significantly increased miR-34a (Figure 6E). However, analysis of AEC lysates for cleaved caspase-3 indicated that neither exposure of PAI-1-deficient mice to BLM nor AEC-specific transduction of Pre-miR-34a in these mice induced apoptosis despite increase in baseline p53 levels (Figure 6G). Furthermore, analysis of lung tissues for fibrosis (Figure 6, H and I) 21 days after transduction of mice with Pre-miR-34a indicated that forced expression of miR-34a did not induce collagen or other matrix protein deposition. Similarly, analyses of lung homogenates for total hydroxyproline (Figure $6 \mathrm{~J}$ ) or collagen (Figure 6K) show that Pre-miR-34a transduction failed to induce PF in PAI-1-deficient mice. This was confirmed by immunoblotting of lung homogenates for Col-1, FN, $\alpha$-SMA, or TN-C (Figure 6L).

\section{miR-34a ${ }^{\mathrm{cKO}}$ Mice Resist BLM-Induced AEC Apoptosis and Development of PF}

We next subjected miR-34a ${ }^{\mathrm{f} / \mathrm{fl}}$ mice transduced with Lv-Cre to BLM injury to determine whether blockade of miR-34a expression inhibits p53 and reverses p53-mediated changes in the uPA-fibrinolytic system and apoptosis in AECs. Consistent with BLM injury in WT mice, AECs of miR-34a ${ }^{\mathrm{f} / \mathrm{fl}}$ mice increased expression of miR-34a after exposure to BLM. This was resisted by $\mathrm{miR}-34 \mathrm{a}^{\mathrm{f} / \mathrm{fl}}$ mice transduced with Lv-Cre (Figure 7A). Analysis of AEC lysates revealed that inhibition of miR-34a expression protected miR-34a ${ }^{\mathrm{f} / \mathrm{fl}}$ mice from BLM-induced apoptosis. Furthermore, AECs showed minimal ${ }^{A c} \mathrm{p} 53$ and total p53, signifying that miR-34a-mediated acetylation contributes to p53 increased steady-state expression (Figure 7B). Consistent with low p53 expression, BLM failed to induce PAI-1 or suppress UPA and uPAR in miR-34a ${ }^{\mathrm{f} / \mathrm{fl}}$ mice treated with Lv-Cre. As expected, AECs of control miR-34a ${ }^{\mathrm{f} / / \mathrm{fl}}$ mice transduced with Lv-Ev responded to BLM injury with induction of $\mathrm{p} 53$ and PAI-1 with synchronized reduction in uPA and uPAR. In accordance with protein expression in AECs, BLM failed to induce PAI-1 or suppress UPA or UPAR mRNAs (Figure $7 \mathrm{C}$ ) in miR-34a $\mathrm{a}^{\mathrm{f} / \mathrm{fl}}$ mice transduced with Lv-Cre, whereas Lv-Ev treated control mice succumbed to BLM injury. Analysis of whole lung homogenates 21 days after BLM injury (Figure 7D) confirmed that BLM did not induce total hydroxyproline contents in $\operatorname{miR}-34 \mathrm{a}^{\mathrm{fl} / \mathrm{fl}}$ mice transduced with Lv-Cre, suggesting protection against lung fibrosis. Western blotting of lung homogenates of miR-34 $\mathrm{a}^{\mathrm{f} / \mathrm{ft}}$ mice exposed to Lv-Cre showed negligible increases in Col-1, FN, $\alpha$-SMA, and TN-C expression (Figure 7E). Further analyses of Col-1, FN, $\alpha$-SMA, and TN-C mRNAs in 
A

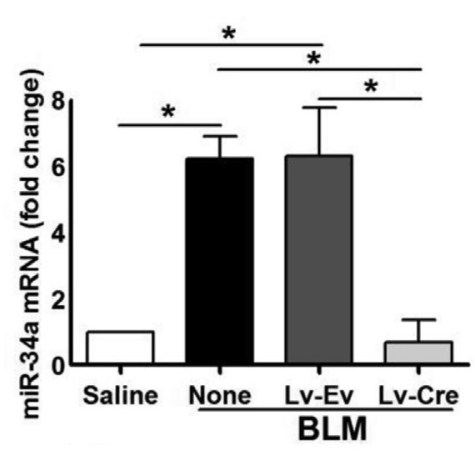

D

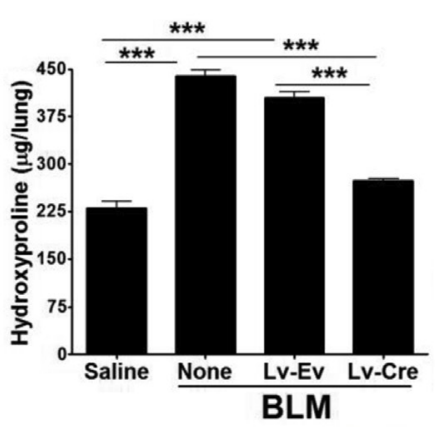

G
B

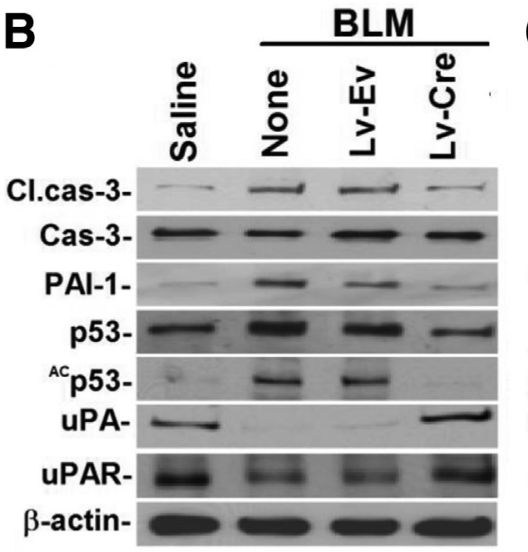

UPAR-

$\beta$-actin-

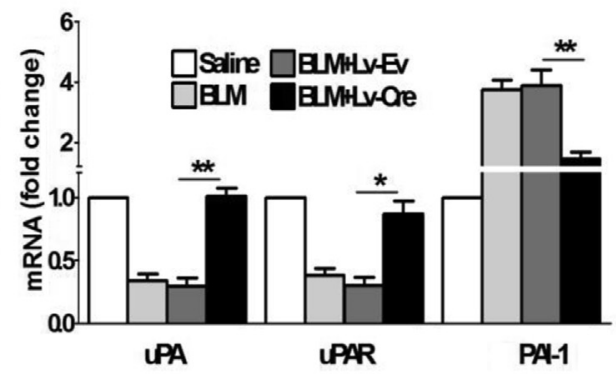

$\mathbf{F}$

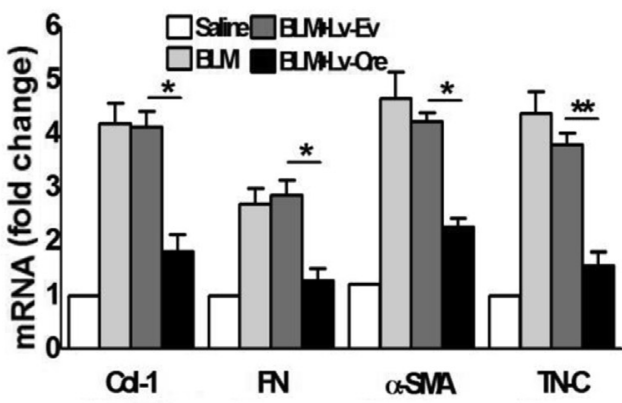

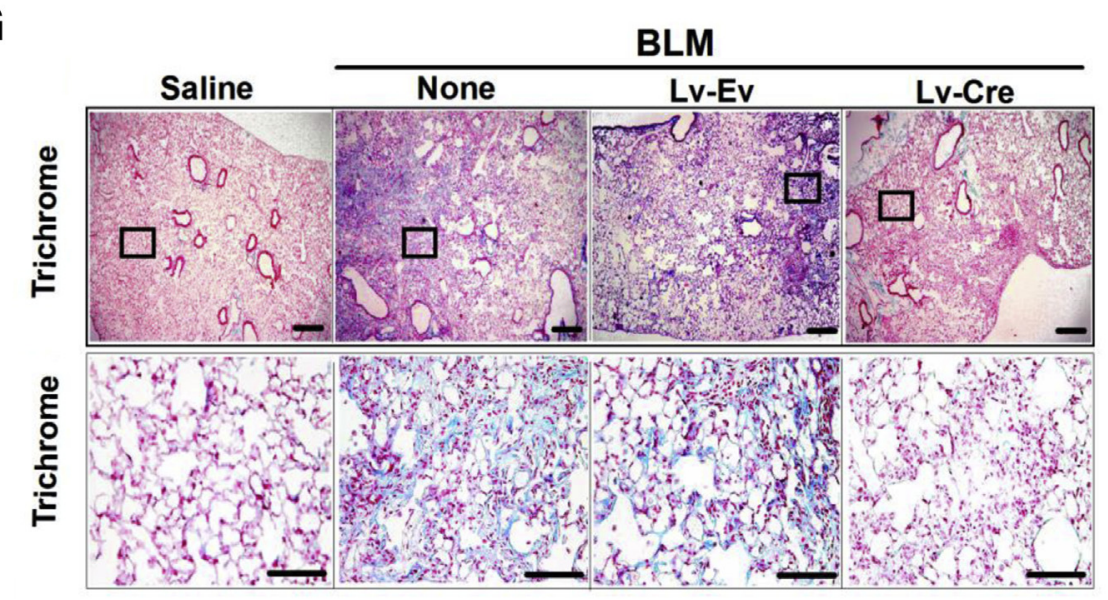

H

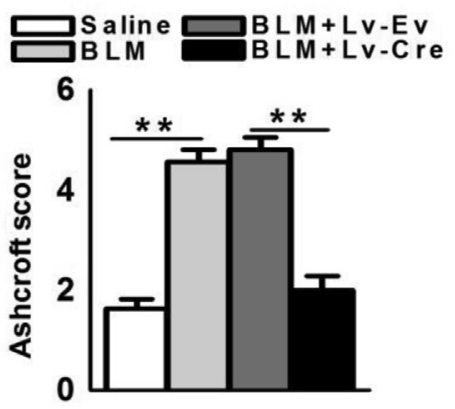

Figure 7 Inhibition of miR-34a expression mitigates bleomycin (BLM)-induced lung epithelial injury and prevents lung fibrosis. A: Alveolar epithelial cells (AECS) were isolated from saline- or BLM-treated miR-34a $\mathrm{a}^{\mathrm{fl} / \mathrm{fl}}$ mice, or BLM-treated miR-34a $\mathrm{a}^{\mathrm{fl} / \mathrm{fl}}$ mice transduced with Lv-Ev or Lv-Cre 3 days after exposure of BLM. RNA from AECs of these mice were analyzed for miR-34a by quantitative real-time RT-PCR. B: AEC lysates from saline- or BLM-treated miR-34a ${ }^{\mathrm{fl} / \mathrm{fl}}$ mice, or miR-34a ${ }^{\mathrm{fl} / \mathrm{fl}}$ mice transduced with Lv-Ev or Lv-Cre and exposed to BLM for 3 days were immunoblotted for ${ }^{A c} \mathrm{p} 53 / \mathrm{p} 53$, PAI-1, uPA, uPAR, cleaved (Cl.)/total caspase-3, and $\beta$-actin. C: RNA from AECs of mice treated as in A was subjected to quantitative real-time RT-PCR for changes in uPA, uPAR, and PAI-1 mRNA. The values were normalized to the corresponding level of $\beta$-actin mRNA in each sample. D: miR- $34 a^{\mathrm{fl} / \mathrm{fl}}$ mice exposed to saline, BLM, or miR-34a $\mathrm{a}^{\mathrm{fl} / \mathrm{fl}}$ mice exposed to Lv-Ev or Lv-Cre treated with BLM were euthanized 21 days after BLM injury. Lung homogenates from these mice were analyzed for hydroxyproline content. E: Lung homogenates from the miR-34a ${ }^{\mathrm{fl} / \mathrm{fl}}$ mice exposed to saline or BLM and Lv-Ev- or Lv-Cre-treated miR ${ }^{\mathrm{fl} / \mathrm{fl}}$ mice as in $\mathbf{D}$ were immunoblotted for Col-1, FN, $\alpha$-SMA, TN-C, and $\beta$-actin. F: RNA from lung tissues of saline, BLM-, Lv-Ev + BLM-, or Lv-Cre + BLM-treated miR ${ }^{\mathrm{fl} / \mathrm{fl}}$ mice 21 days after BLM injury as in $\mathbf{D}$ were analyzed for Col-1, FN, $\alpha$-SMA, and TN-C mRNA by quantitative real-time RT-PCR and were normalized against corresponding levels of $\beta$-actin mRNA. G: Lung sections of miR34 $4^{\mathrm{fl} f \mathrm{fl}}$ mice treated with saline, BLM, Lv-Ev + BLM, or Lv-Cre + BLM as in A and euthanized 21 days after BLM injury were subjected to trichrome staining. Boxed areas are shown at higher magnification in the bottom row. H: Bar graph represents morphometric quantification of PF by Ashcroft scoring. Data are expressed as means \pm SD. Each experiment was repeated at least three times. $n=6$ per group $(\mathbf{A}$ and $\mathbf{B})$. ${ }^{*} P<0.05,{ }^{* *} P<0.01$, and ${ }^{* * *} P<0.001$. Scale bars: $300 \mu \mathrm{m}$ (G, top row); $100 \mu \mathrm{m}$ (G, bottom row). Original magnification: $\times 4$ (G, top row); $\times 20$ (G, bottom row). 
A B

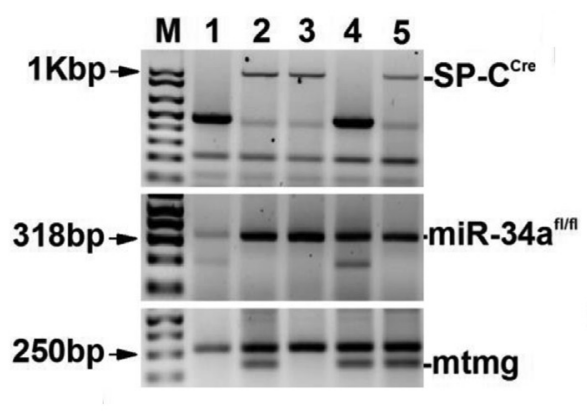

B

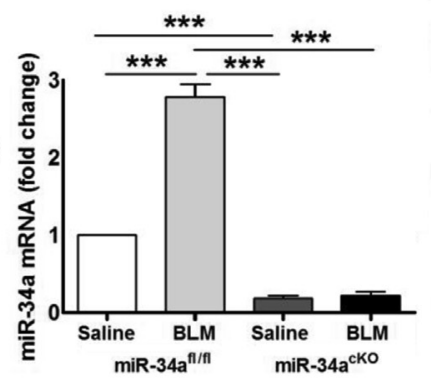

Cl.cas-3-

Cas-3-

PAl-1-

UPA-

uPAR-

Sirt-1-

$\beta$-actin-

p53-

$\beta$-actin-
D

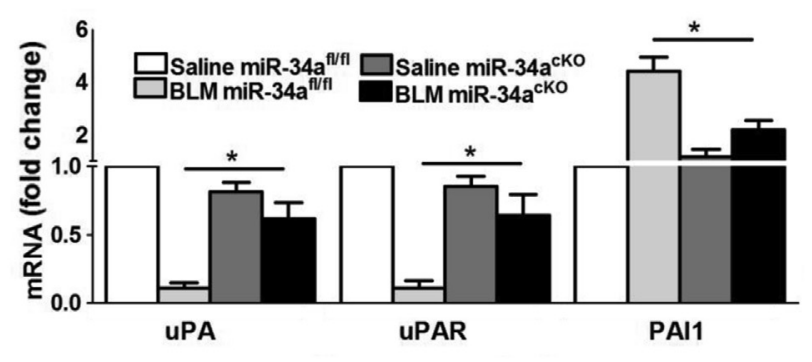

$\mathbf{F}$

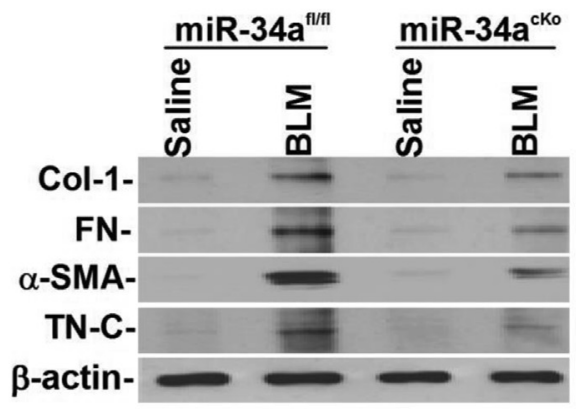

G
E

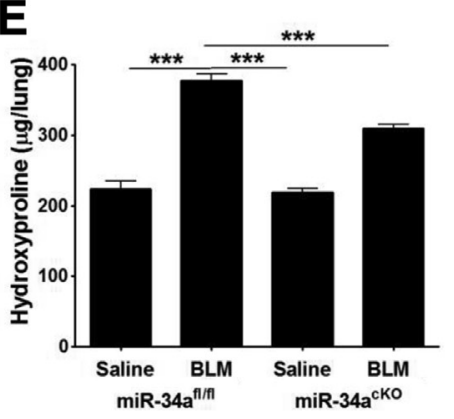

H
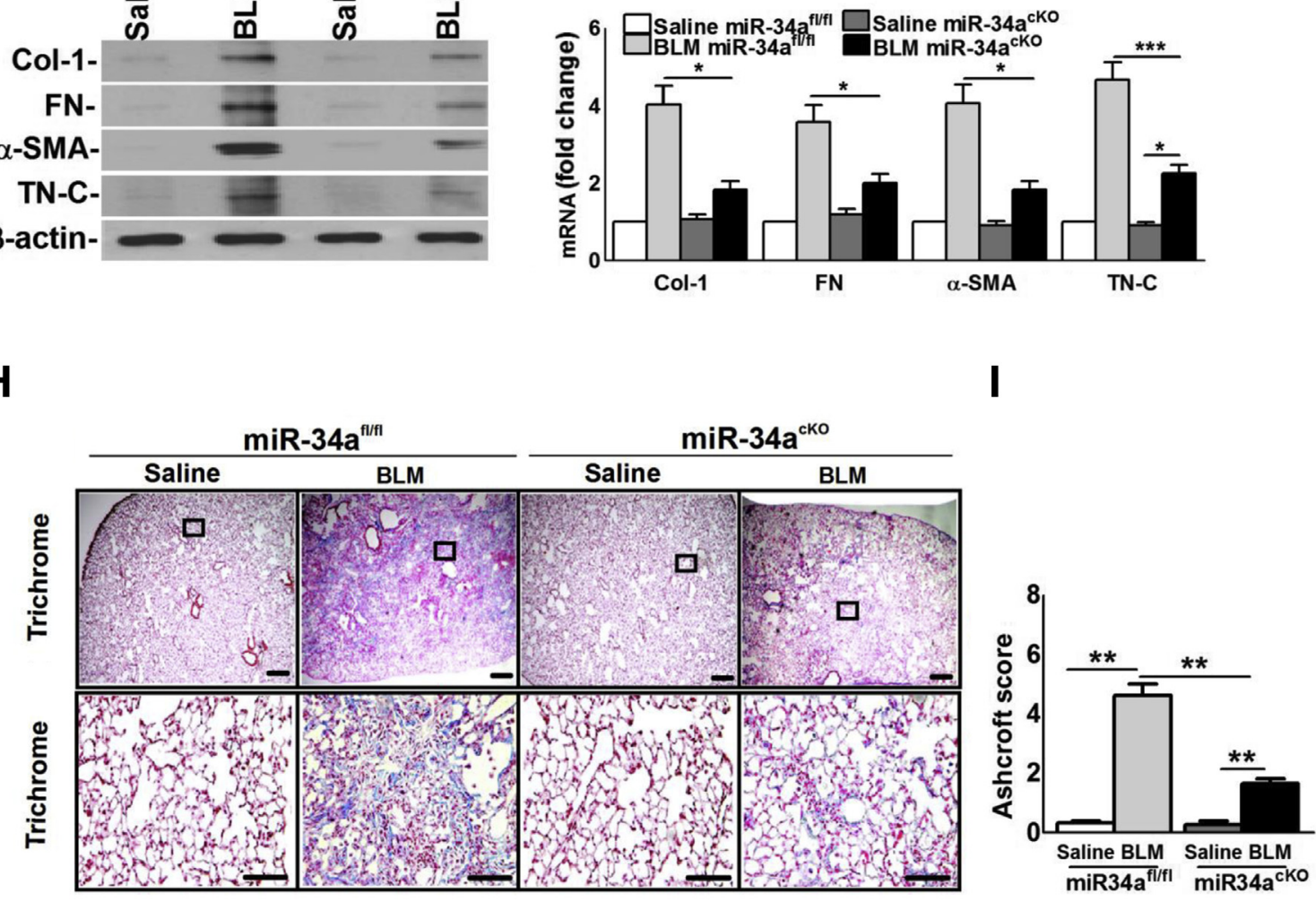
these mice (Figure 7F) also proved minimal fibrogenesis after suppression of BLM-induced miR-34a. Trichrome staining (Figure $7 \mathrm{G}$ ) and morphometric analysis (Figure $7 \mathrm{H}$ ) of lung sections of miR-34 $\mathrm{a}^{\mathrm{f} / \mathrm{fl}}$ mice exposed to BLM or BLM and $\mathrm{Lv}$-Ev displayed excessive collagen and other matrix protein deposits, which were markedly reduced after blockade of miR-34a expression using Lv-Cre.

We generated miR-34a $\mathrm{a}^{\mathrm{fl}} / \mathrm{SP}-\mathrm{C}^{\mathrm{Cre} / \mathrm{mTmG}}$ mice (Figure $8 \mathrm{~A}$ ) by cross-breeding miR-34a $\mathrm{a}^{\mathrm{f} / \mathrm{fl}}$ with $\mathrm{SP}-\mathrm{C}^{\mathrm{Cre} / \mathrm{mTmG}}$ mice. These mice were i.p. injected with tamoxifen to induce Cre recombinase for suppression of miR-34a expression in AECs. To determine whether increased miR-34a expression in AECs contributes to apoptosis in vivo, inducible miR-34a $\mathrm{a}^{\mathrm{cKO}}$ mice lacking miR-34a expression in AECs were exposed to BLM. BLM failed to increase AEC miR-34a expression in miR-34a ${ }^{\mathrm{cKO}}$ mice, whereas it induced its expression in miR$34 \mathrm{a}^{\mathrm{fl} / \mathrm{fl}}$ mice (Figure 8B). Immunoblotting of AEC lysates revealed that BLM failed to inhibit UPA or UPAR, or induce robust p53 or PAI-1 expression or apoptosis in miR-34a $\mathrm{a}^{\mathrm{cKO}}$ mice (Figure $8 \mathrm{C}$ ). RNA analysis of AECs from miR-34a $\mathrm{a}^{\mathrm{cKO}}$ mice demonstrated little change in UPA, UPAR, and PAI-1 mRNA expression after exposure to BLM (Figure 8D), indicating lack of p53-uPA system cross talk. To determine whether induction of miR-34a in AECs contributes to the development of PF, we analyzed the whole lung homogenates of miR-34a ${ }^{\mathrm{cKO}}$ mice for total hydroxyproline contents. miR-34a ${ }^{\mathrm{cKO}}$ mice exposed to BLM developed significantly less PF compared to WT or miR-34 $\mathrm{a}^{\mathrm{t} / \mathrm{fl}}$ mice with BLM injury because of loss of AEC-specific miR-34a expression (Figure 8E). Immunoblotting of lung homogenates also showed minimal increases in Col-1, FN, $\alpha$-SMA, and TN-C protein (Figure $8 \mathrm{~F}$ ) or mRNA (Figure 8G) in the lung tissues of miR-34a $a^{\mathrm{cKO}}$ mice treated with BLM. The resistance of miR-34a ${ }^{\text {cKO }}$ mice to BLM-induced PF was further validated by analyses of the lung tissues (Figure 8, H and I), which showed minimal collagen and other matrix deposits, indicating that increased AEC expression of miR-34a contributes to the development of PF.

\section{Discussion}

IPF affects 500,000 people in the United States and Europe per year, with a median survival of $<2$ to 3 years after diagnosis. ${ }^{49,50}$ A hallmark of IPF is the formation of fibroblastic foci, consisting of large numbers of fibrotic lung fibroblasts and interstitial fibrosis with architectural distortion. Characteristic morphological lesions include spatial and temporal heterogeneity incorporating areas of normal lung adjacent to areas of evolving fibrosis containing fibrotic lung fibroblasts. Increased expression of p53, PAI-1, and apoptosis are found in AECs of interstitial lung diseases, including human IPF and in animal models of PF. ${ }^{22}$ Cell cycle arrest, senescence, or apoptosis of AECs involves suppression of survival signals with increased p53 expression observed in BLM-, silica-, or CS-exposureinduced lung injury. ${ }^{10-12,14}$

We reported elevated levels of apoptotic AECs in IPF lungs and mice with established PF. ${ }^{12,22}$ Further transplantation of healthy AECs into the lungs of mice with $\mathrm{BLM}^{28}{ }^{28}$ or silica- ${ }^{29}$ induced lung injury inhibits PF. These findings buttress the concept that AEC apoptosis and injury leads to development of $\mathrm{PF}$ and of the idea that preservation of AEC viability protects against subsequent PF. After activation, p53 controls target genes involved in the regulation of cell cycle, quiescence, senescence, differentiation, and cell apoptosis, including uPA, uPAR, and PAI-1. Along these lines, genetic loss of p53 or PAI-1 in mice has been associated with resistance to BLM-, CS-, or silicaexposure-induced AEC apoptosis and PF. ${ }^{11,12,22}$ Loss of uPA increases p53 and PAI-1 expression, and apoptosis in AECs and fibrogenesis. ${ }^{12,13,20}$

p53 is tightly regulated and usually expressed at low levels in cells. Basal expression of p53 is naturally maintained at low levels by targeted degradation via $\mathrm{mdm} 2{ }^{44}$ The importance of $\mathrm{mdm} 2$ in regulating low basal expression of p53 is best illustrated by the findings that deletion of mdm2 leads to elevated p53 levels and cellular lethality as well as abnormalities in various organs and impaired survival even in the absence of DNA damage. ${ }^{51}$ Furthermore, complete rescue of the embryonic lethality because of loss of $\mathrm{mdm} 2$ after depletion or inactivation of $\mathrm{p} 53^{52}$ underlines the pathophysiological significance of $\mathrm{mdm} 2$-mediated regulation of $\mathrm{p} 53$. These observations imply that excessive p53 accumulation stalls proliferation of cells leading to organ damage, whereas the loss of p53 increases tumorigenesis. The balance between $\mathrm{p} 53$ and $\mathrm{mdm} 2$ in the maintenance of tissue homeostasis is underlined by the

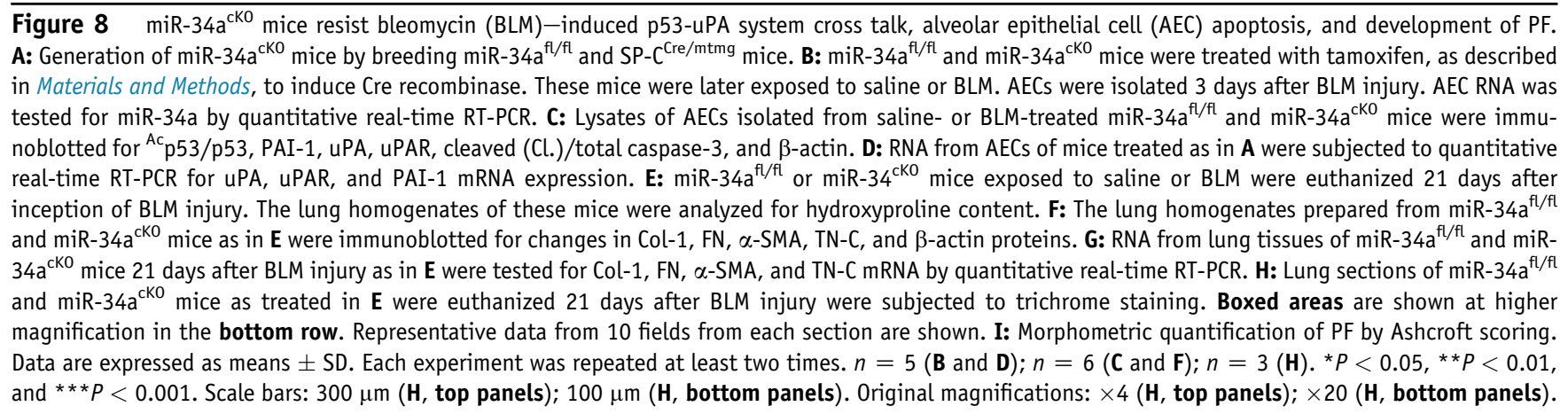


sensitivity of actively proliferating cells to activation of p53 via loss of mdm2.

p53 levels are rapidly and markedly increased through protein stabilization in response to various cellular stresses. The process involves posttranslational modifications, such as phosphorylation at serine residues and acetylation of lysine residues of $\mathrm{p} 53$, which prevent interactions of $\mathrm{p} 53$ with negative regulators $\mathrm{mdm} 2$ and $\mathrm{mdmx}^{44}$ and protect against degradation of $\mathrm{p} 53$, leading to its stabilization. ${ }^{54}$ Lack of interaction of mdm2 with the acetylated p53 is independent of its phosphorylation status ${ }^{55}$ and increased acetylation of $\mathrm{p} 53$ promotes its steady-state expression. This concept is validated by the dramatic loss of growth arrest and apoptosis functionality of acetylation-defective p53 and the restoration of function of unacetylated p53 after loss of mdm2. ${ }^{56}$ Besides acetylation by acetylases/acetyl transferases, broad class I/II histone deacetylase inhibitor, trichostatin A, and a specific small-molecule Sirt1 catalytic activity inhibitor (EX-527) increase acetylation of p53, whereas overexpression of Sirt $1^{57}$ reduces acetylated and steady-state levels of p53.

Increased lysine 379 acetylation of p53 in AECs isolated from injured lungs suggests that posttranslational modification contributes to increased p53 expression. Consistent with p53 acetylation, Sirt1 is reduced with increased miR-34a in AECs isolated from injured lungs, which is reversed by CSP. Sirtl knockdown sensitizes cells to apoptosis and reduces cell survival. ${ }^{58}$ Sirt1 has been implicated in deacetylation of p53, ${ }^{59}$ and miR-34a inhibits Sirt1 translation, ${ }^{59,60}$ indicating that miR-34a-mediated suppression of Sirt1 expression contributes to the accumulation of ${ }^{\mathrm{Ac}} \mathrm{p} 53$ and total p53. Consistent with these findings, increased miR-34a, p53, and PAI-1 expression, and apoptosis occurs with marked suppression of Sirt1 in AECs isolated from IPF lungs or from the lungs of mice with BLM-, silica-, CS-, or sepsis-induced lung injury. Furthermore, inhibition of miR-34a expression in injured AECs reduces p53, PAI-1, and apoptosis. On the contrary, overexpression of miR-34a in AECs augments apoptosis with increases in $\mathrm{p} 53$ and PAI-1, indicating that miR-34a/Sirt1mediated control of acetylation and stabilization of p53 plays a pivotal role in various lung injuries.

p53 concurrently induces PAI- 1 and suppresses uPA both at the transcriptional and posttranscriptional levels, ${ }^{11,47,48,61}$ while suppressing uPAR expression through mRNA destabilization. ${ }^{13,62}$ Targeting p53-mediated downstream induction of PAI-1 and suppression of UPA and UPAR increases AEC viability, ${ }^{11,13}$ demonstrating the consequences of AEC-specific p53-uPA system cross talk in lung injury and fibrotic remodeling. Furthermore, forced expression of miR-34a in AECs induced PF in mice 21 days later, indicating that miR-34a-mediated AEC apoptosis contributes to subsequent fibrogenesis. CSP did not mitigate BLMinduced miR-34a expression in mice lacking uPA. This is consistent with the inability of CSP to suppress p53 or AEC apoptosis or the development of PF in uPA-deficient mice. ${ }^{12,20}$ Interestingly, inhibition of miR-34a in AECs suppressed BLM-induced p53 and PAI-1 expression, and apoptosis in uPA-deficient mice. These mice also resisted BLM-induced PF. We believe that slightly elevated levels of hydroxyproline content found in the lungs of uPA-deficient mice exposed to BLM compared to similarly treated WT mice may be attributed to increased susceptibility of mice lacking uPA expression to BLM-induced lung epithelial injury and subsequent PF, as previously reported. ${ }^{12,13,63,64}$ p53-deficient mice exposed to BLM failed to induce miR34a expression in AECs, apoptosis, or PF. This is consistent with augmented AEC p53 and PAI-1 expression, and apoptosis, and PF in WT and uPA-deficient mice exposed to BLM, and suppression of epithelial injury, PAI-1 expression, and fibrogenesis after blockade of BLM-induced p53 from binding to AEC uPA, uPAR, and PAI-1 mRNAs in these mice. ${ }^{13}$ In addition, miR-34a is a transcriptional target of p53, suggesting a positive feedback regulation exists between miR-34a and p53, in part through p53-uPA system cross talk. Taken together, this study indicates that p53 and miR-34a through autoregulatory feedback induction controls AEC apoptosis and subsequent development of PF, and that the process requires suppression of uPA. In addition, lack of miR-34a induction, AEC apoptosis, and PF in PAI-1-deficient mice also suggests that uPA turnover by the ternary complex uPA-uPAR-PAI-1 contributes to p53-miR-34a feed forward induction. Although p53 is induced in AECs of PAI-1-deficient mice, both p53- and PAI-1-deficient mice resist PF because of lack of PAI-1 induction. ${ }^{22}$ Significantly higher levels of fibrotic markers observed in the lung homogenates of PAI-1-deficient mice are consistent with our earlier report ${ }^{20}$ and are attributable to increased expression of these proteins by AECs, probably as a safeguard to protect against uncontrolled AEC apoptosis.

Although the importance of p53 acetylation in relation to its function remains controversial, ${ }^{36}$ acetylation is invariably associated with increased p53 protein abundance, DNA binding, and activation of target genes through stabilization of p53 protein. ${ }^{55}$ However, the effect of acetylation on RNA binding by $\mathrm{p} 53$ has not been established. We found that $\mathrm{p} 53$ concurrently binds to UPA, uPAR, and PAI- 1 mRNA $3^{\prime}$ untranslated region determinants through its C-terminal domain (amino acids 296 to 393). ${ }^{48}$ We also reported that BLM injury augments p53 protein binding to endogenous uPA, uPAR, and PAI-1 mRNA $3^{\prime}$ untranslated regions in AECs. ${ }^{13}$ Our new data reveal that both chronic and acute lung injuries augment p53 acetylation at residue 379 in AECs. Therefore, we presume that acetylated p53 binds to target uPA, uPAR, and PAI-1 mRNA.

In addition, p53 can directly bind to the PAI-1 (Serpine1) promoter and induce PAI-1 mRNA transcription while inhibiting uPA mRNA synthesis by affecting the function of specific transcription factors that control uPA mRNA synthesis. ${ }^{47}$ Because modifications such as ${ }^{\mathrm{p} 15 \mathrm{~S}} \mathrm{p} 53$ and acetylation of lysine 379 residues of p53 are linked to p53 expression and function, and ${ }^{\mathrm{p} 15 \mathrm{~S}} \mathrm{p} 53$ itself can undergo 
autoacetylation, we believe that acetylation of $\mathrm{p} 53$ regulates uPA, uPAR, and PAI-1 at the transcriptional and posttranscriptional levels. The resistance of $\mathrm{miR}-34 \mathrm{a}^{\mathrm{t} / \mathrm{fl}}$ mice transduced with LV-Cre or miR-34 $\mathrm{a}^{\mathrm{cKO}}$ mice lacking miR34a expression in AECs to BLM-induced p53 and PAI-1 expression or apoptosis in AECs, and development of lung fibrosis underlines the importance of increased miR34a in the pathogenesis of fibrosing lung injuries.

p53 induces miR-34a transcription while miR-34a augments acetylation of p53 through inhibition of Sirt1. This leads to stabilization of p53 in AECs in the chronic and acute lung injuries we studied. p53 inhibits uPA and UPAR while inducing PAI-1 expression, whereas uPA and UPAR inhibit p53 and downstream PAI-1 expression. Inhibition of UPA and UPAR expression and induction of PAI-1 expression are correlated with increased ${ }^{A c} \mathrm{p} 53$ and miR-34a-mediated suppression of Sirt1 in AECs. Together, these changes induce AEC apoptosis and PF, which can be reversed by CSP. Suppression of miR-34a also inhibits apoptosis and prevents PF. The process involves cell surface signaling involving caveolin-1, uPAR, $\beta 1$-integrin, and Src kinase. ${ }^{12}$ Our study reveals that miR-34a via its ability to induce p53 and changes in the uPA-fibrinolytic system causes apoptosis in AECs and PF. As miR-34a-mediated acetylation of p53 is reversible, we infer that this pathway can be targeted to mitigate AEC injury and PF.

\section{Acknowledgments}

We thank Dr. Hal Chapman (University of California, San Francisco, CA) for providing breeding pairs of SP-C $\mathrm{C}^{\mathrm{Cre} / \mathrm{mTmG}}$ mice.

S.K.S., N.T., A.S.M., B.P., and Y.P.B. performed the experiments and analyzed the data; J.F. and J.L. provided unique reagents and analyzed data; S.I. analyzed data and pathology and edited the manuscript; S.S. conceived and designed the experiments and wrote the manuscript.

\section{References}

1. Lakin ND, Jackson SP: Regulation of p53 in response to DNA damage. Oncogene 1999, 18:7644-7655

2. Sui X, Han W, Pan H: p53-Induced autophagy and senescence. Oncotarget 2015, 6:11723-11724

3. Granja AG, Nogal ML, Hurtado C, Salas J, Salas ML, Carrascosa AL, Revilla Y: Modulation of p53 cellular function and cell death by African swine fever virus. J Virol 2004, 78:7165-7174

4. Lowe SW, Schmitt EM, Smith SW, Osborne BA, Jacks T: p53 Is required for radiation-induced apoptosis in mouse thymocytes. Nature 1993, 362:847-849

5. Felton-Edkins ZA, Kenneth NS, Brown TRP, Daly NL, Gomez-Roman N, Grandori C, Eisenman RN, White RJ: Direct regulation of RNA polymerase III transcription by RB, p53 and c-Myc. Cell Cycle 2003, 2:180-183

6. Tarasov V, Jung P, Verdoodt B, Lodygin D, Epanchintsev A, Menssen A, Meister G, Hermeking H: Differential regulation of microRNAs by p53 revealed by massively parallel sequencing:
miR-34a is a p53 target that induces apoptosis and G1-arrest. Cell Cycle 2007, 6:1586-1593

7. Xi Y, Shalgi R, Fodstad O, Pilpel Y, Ju J: Differentially regulated micro-RNAs and actively translated messenger RNA transcripts by tumor suppressor p53 in colon cancer. Clin Cancer Res 2006, 12: 2014-2024

8. Werner H, Karnieli E, Rauscher FJ, LeRoith D: Wild-type and mutant p53 differentially regulate transcription of the insulin-like growth factor I receptor gene. Proc Natl Acad Sci 1996, 93:8318-8323

9. Huarte M, Guttman M, Feldser D, Garber M, Koziol MJ, Kenzelmann-Broz D, Khalil AM, Zuk O, Amit I, Rabani M, Attardi LD, Regev A, Lander ES, Jacks T, Rinn JL: A large intergenic noncoding RNA induced by p53 mediates global gene repression in the p53 response. Cell 2010, 142:409-419

10. Bhandary YP, Shetty SK, Marudamuthu AS, Midde KK, Ji H-L, Shams H, Subramaniam R, Fu J, Idell S, Shetty S: Plasminogen activator inhibitor-1 in cigarette smoke exposure and influenza A virus infection-induced lung injury. PLoS One 2015, 10:e0123187

11. Shetty SK, Bhandary YP, Marudamuthu AS, Abernathy D, Velusamy T, Starcher B, Shetty S: Regulation of airway and alveolar epithelial cell apoptosis by p53-induced plasminogen activator inhibitor-1 during cigarette smoke exposure injury. Am J Respir Cell Mol Biol 2012, 47:474-483

12. Bhandary YP, Shetty SK, Marudamuthu AS, Gyetko MR, Idell S, Gharaee-Kermani M, Shetty RS, Starcher BC, Shetty S: Regulation of alveolar epithelial cell apoptosis and pulmonary fibrosis by coordinate expression of components of the fibrinolytic system. Am J Physiol Lung Cell Mol Physiol 2012, 302:L463-L473

13. Bhandary YP, Shetty SK, Marudamuthu AS, Ji H-L, Neuenschwander PF, Boggaram V, Morris GF, Fu J, Idell S, Shetty S: Regulation of lung injury and fibrosis by p53-mediated changes in urokinase and plasminogen activator inhibitor-1. Am J Pathol 2013, 183:131-143

14. Bhandary YP, Shetty SK, Marudamuthu AS, Fu J, Pinson BM, Levin J, Shetty S: Role of p53-fibrinolytic system cross-talk in the regulation of quartz-induced lung injury. Toxicol Appl Pharmacol 2015, 283:92-98

15. Hickman AW, Jaramillo RJ, Lechner JF, Johnson NF: $\alpha$-Particleinduced p53 protein expression in a rat lung epithelial cell strain. Cancer Res 1994, 54:5797-5800

16. O'Reilly MA, Staversky RJ, Stripp BR, Finkelstein JN: Exposure to hyperoxia induces p53 expression in mouse lung epithelium. Am J Respir Cell Mol Biol 1998, 18:43-50

17. Mantell LL, Lee PJ: Signal transduction pathways in hyperoxiainduced lung cell death. Mol Genet Metab 2000, 71:359-370

18. Sermeus A, Michiels C: Reciprocal influence of the p53 and the hypoxic pathways. Cell Death Dis 2011, 2:e164

19. Sutherland LM, Edwards YS, Murray AW: Alveolar type II cell apoptosis. Comp Biochem Physiol A Mol Integr Physiol 2001, 129: 267-285

20. Marudamuthu AS, Bhandary YP, Shetty SK, Fu J, Sathish V, Prakash Y, Shetty S: Role of the urokinase-fibrinolytic system in epithelial-mesenchymal transition during lung injury. Am J Pathol 2015, 185:55-68

21. Nakashima N, Kuwano K, Maeyama T, Hagimoto N, Yoshimi M, Hamada N, Yamada M, Nakanishi Y: The p53-Mdm2 association in epithelial cells in idiopathic pulmonary fibrosis and non-specific interstitial pneumonia. J Clin Pathol 2005, 58:583-589

22. Marudamuthu AS, Shetty SK, Bhandary YP, Karandashova S, Thompson M, Sathish V, Florova G, Hogan TB, Pabelick CM, Prakash YS, Tsukasaki Y, Fu J, Ikebe M, Idell S, Shetty S: Plasminogen activator inhibitor-1 suppresses profibrotic responses in fibroblasts from fibrotic lungs. J Biol Chem 2015, 290:9428-9441

23. Morse D, Rosas IO: Tobacco smoke-induced lung fibrosis and emphysema. Annu Rev Physiol 2014, 76:493-513

24. Mitchell PD, Das JP, Murphy DJ, Keane MP, Donnelly SC, Dodd JD, Butler MW: Idiopathic pulmonary fibrosis with emphysema: evidence 
of synergy among emphysema and idiopathic pulmonary fibrosis in smokers. Respir Care 2015, 60:259-268

25. Burnham EL, Janssen WJ, Riches DWH, Moss M, Downey GP: The fibroproliferative response in acute respiratory distress syndrome: mechanisms and clinical significance. Eur Respir J 2014, 43: 276-285

26. Martin C, Papazian L, Payan M-J, Saux P, Gouin F: Pulmonary fibrosis correlates with outcome in adult respiratory distress syndrome: a study in mechanically ventilated patients. Chest 1995, 107: $196-200$

27. Uhal BD, Joshi I, Hughes WF, Ramos C, Pardo A, Selman M: Alveolar epithelial cell death adjacent to underlying myofibroblasts in advanced fibrotic human lung. Am J Physiol Lung Cell Mol Physiol 1998, 275:L1192-L1199

28. Serrano-Mollar A, Nacher M, Gay-Jordi G, Closa D, Xaubet A, Bulbena O: Intratracheal transplantation of alveolar type II cells reverses bleomycin-induced lung fibrosis. Am J Respir Crit Care Med 2007, 176:1261-1268

29. Spitalieri P, Quitadamo MC, Orlandi A, Guerra L, Giardina E, Casavola V, Novelli G, Saltini C, Sangiuolo F: Rescue of murine silica-induced lung injury and fibrosis by human embryonic stem cells. Eur Respir J 2012, 39:446-457

30. Feng Z, Zhang $\mathrm{C}$, Wu R, Hu W: Tumor suppressor p53 meets microRNAs. J Mol Cell Biol 2011, 3:44-50

31. Hermeking H: p53 Enters the microRNA world. Cancer Cell 2007, $12: 414-418$

32. Felekkis K, Touvana E, Stefanou C, Deltas C: microRNAs: a newly described class of encoded molecules that play a role in health and disease. Hippokratia 2010, 14:236-240

33. Wilczynska A, Bushell M: The complexity of miRNA-mediated repression. Cell Death Differ 2015, 22:22-33

34. Raver-Shapira N, Marciano E, Meiri E, Spector Y, Rosenfeld N, Moskovits N, Bentwich Z, Oren M: Transcriptional activation of miR-34a contributes to p53-mediated apoptosis. Mol Cell 2007, 26: $731-743$

35. Fridman JS, Lowe SW: Control of apoptosis by p53. Oncogene 2003, 22:9030-9040

36. Solomon JM, Pasupuleti R, Xu L, McDonagh T, Curtis R, DiStefano PS, Huber LJ: Inhibition of SIRT1 catalytic activity increases p53 acetylation but does not alter cell survival following DNA damage. Mol Cell Biol 2006, 26:28-38

37. Kanegai CM, Xi Y, Donne ML, Gotts JE, Driver IH, Amidzic G, Lechner AJ, Jones KD, Vaughan AE, Chapman HA, Rock JR: Persistent pathology in influenza-infected mouse lungs. Am J Respir Cell Mol Biol 2016, 55:613-615

38. Gao R, Ma Z, Hu Y, Chen J, Shetty S, Fu J: Sirt1 restrains lung inflammasome activation in a murine model of sepsis. Am J Physiol Lung Cell Mol Physiol 2015, 308:L847-L853

39. Chang J, Nicolas E, Marks D, Sander C, Lerro A, Buendia MA, Xu C, Mason WS, Moloshok T, Bort R, Zaret KS, Taylor JM: miR122, a mammalian liver-specific microRNA, is processed from hcr mRNA and may downregulate the high affinity cationic amino acid transporter CAT-1. RNA Biol 2004, 1:106-113

40. Shetty S, Kumar A, Idell S: Posttranscriptional regulation of urokinase receptor mRNA: identification of a novel urokinase receptor mRNA binding protein in human mesothelioma cells. Mol Cell Biol 1997, 17:1075-1083

41. Tiwari N, Marudamuthu AS, Tsukasaki Y, Ikebe M, Fu J, Shetty S: p53- and PAI-1-mediated induction of C-X-C chemokines and CXCR2: importance in pulmonary inflammation due to cigarette smoke exposure. Am J Physiol Lung Cell Mol Physiol 2016, 15: L496-L506

42. Ashcroft T, Simpson JM, Timbrell V: Simple method of estimating severity of pulmonary fibrosis on a numerical scale. J Clin Pathol 1988, 41:467-470

43. Hübner R-H, Gitter W, El Mokhtari NE, Mathiak M, Both M, Bolte H, Freitag-Wolf S, Bewig B: Standardized quantification of pulmonary fibrosis in histological samples. Biotechniques 2008, 44 507-515

44. Zhao B, Chen H, Lei N, Li G, Zhao W, Zhan Y, Liu B, Lin S, Wu Q: p53 Mediates the negative regulation of MDM2 by orphan receptor TR3. EMBO J 2006, 25:5703-5715

45. Barlev NA, Liu L, Chehab NH, Mansfield K, Harris KG, Halazonetis TD, Berger SL: Acetylation of p53 activates transcription through recruitment of coactivators/histone acetyltransferases. Mol Cell 2001, 8:1243-1254

46. Hasegawa M, Zhang Y, Niibe H, Terry NH, Meistrich ML: Resistance of differentiating spermatogonia to radiation-induced apoptosis and loss in p53-deficient mice. Radiat Res 1998, 149:263-270

47. Kunz C, Pebler S, Otte J, von der Ahe D: Differential regulation of plasminogen activator and inhibitor gene transcription by the tumor suppressor p53. Nucleic Acids Res 1995, 23:3710-3717

48. Shetty S, Shetty P, Idell S, Velusamy T, Bhandary YP, Shetty RS: Regulation of plasminogen activator inhibitor-1 expression by tumor suppressor protein p53. J Biol Chem 2008, 283:19570-19580

49. Coultas DB, Zumwalt RE, Black WC, Sobonya RE: The epidemiology of interstitial lung diseases. Am J Respir Crit Care Med 1994, 150:967-972

50. Datta A, Scotton CJ, Chambers RC: Novel therapeutic approaches for pulmonary fibrosis. Br J Pharmacol 2011, 163:141-172

51. Valentin-Vega YA, Okano H, Lozano G: The intestinal epithelium compensates for p53-mediated cell death and guarantees organismal survival. Cell Death Differ 2008, 15:1772-1781

52. El-Dahr SS, Hilliard S, Aboudehen K, Saifudeen Z: The MDM2-p53 pathway: multiple roles in kidney development. Pediatr Nephrol 2014, 29:621-627

53. Lozano G: Mouse models of p53 functions. Cold Spring Harb Perspect Biol 2010, 2:a001115

54. Freedman DA, Levine AJ: Nuclear export is required for degradation of endogenous p53 by MDM2 and human papillomavirus E6. Mol Cell Biol 1998, 18:7288-7293

55. Tang Y, Zhao W, Chen Y, Zhao Y, Gu W: Acetylation is indispensable for p53 activation. Cell 2008, 133:612-626

56. Ito A, Lai CH, Zhao X, Saito S, Hamilton MH, Appella E, Yao TP: p300/CBP-mediated p53 acetylation is commonly induced by p53activating agents and inhibited by MDM2. EMBO J 2001, 20: $1331-1340$

57. Yang H, Yan B, Liao D, Huang S, Qiu Y: Acetylation of HDAC1 and degradation of SIRT1 form a positive feedback loop to regulate p53 acetylation during heat-shock stress. Cell Death Dis 2015, 6:e1747

58. Zhao G, Cui J, Zhang J-G, Qin Q, Chen Q, Yin T, Deng S-C, Liu Y, Liu L, Wang B, Tian K, Wang G-B, Wang C-Y: SIRT1 RNAi knockdown induces apoptosis and senescence, inhibits invasion and enhances chemosensitivity in pancreatic cancer cells. Gene Ther 2011, 18:920-928

59. Lee JT, Gu W: SIRT1: regulator of p53 deacetylation. Genes Cancer 2013, 4:112-117

60. Yamakuchi M, Ferlito M, Lowenstein CJ: miR-34a repression of SIRT1 regulates apoptosis. Proc Natl Acad Sci U S A 2008, 105:13421-13426

61. Shetty P, Velusamy T, Bhandary YP, Shetty RS, Liu M-C, Shetty S: Urokinase expression by tumor suppressor protein p53: a novel role in mRNA turnover. Am J Respir Cell Mol Biol 2008, 39:364-372

62. Shetty S, Velusamy T, Idell S, Shetty P, Mazar AP, Bhandary YP, Shetty RS: Regulation of urokinase receptor expression by p53: novel role in stabilization of uPAR mRNA. Mol Cell Biol 2007, 27: $5607-5618$

63. Swaisgood CM, French EL, Noga C, Simon RH, Ploplis VA: The development of bleomycin-induced pulmonary fibrosis in mice deficient for components of the fibrinolytic system. Am J Pathol 2000, 157:177-187

64. Sisson TH, Hanson KE, Subbotina N, Patwardhan A, Hattori N, Simon RH: Inducible lung-specific urokinase expression reduces fibrosis and mortality after lung injury in mice. Am J Physiol Lung Cell Mol Physiol 2002, 283:L1023-L1032 